\title{
Diaphragm-Type Pneumatic-Driven Soft Grippers for Precision Harvesting
}

\author{
Eduardo Navas*(D), Roemi Fernández*(D), Manuel Armada (D) and Pablo Gonzalez-de-Santos (iD \\ Centre for Automation and Robotics, UPM-CSIC, Carretera CAMPO-REAL Km 0.2, Arganda del Rey, \\ 28500 Madrid, Spain; manuel.armada@csic.es (M.A.); pablo.gonzalez@car.upm-csic.es (P.G.-d.-S.) \\ * Correspondence: eduardo.navas@csic.es (E.N.); roemi.fernandez@car.upm-csic.es (R.F.)
}

\begin{abstract}
Soft actuator technology and its role in robotic manipulation have been rapidly gaining ground. However, less attention has been given to the potential advantages of its application to the agricultural sector, where soft robotics may be a game changer due to its greater adaptability, lower cost and simplicity of manufacture. This article presents a new design approach for soft grippers based on modules that incorporate the concept of bellows and combine it with the versatility and replicability of a 3D printed structure. In this way, the modules can be freely configured to obtain grippers adaptable to crops of different diameters. Furthermore, the definition of a method to determine the soft grippers features is also presented, with the aim of serving as the basis for a future benchmarking study on soft actuators. The experimental tests carried out demonstrated the feasibility and capability of the end-effectors to manipulate various fruits, ensuring a sufficient contact area for the safe handling of the targets and avoiding damaging the products.
\end{abstract}

Keywords: harvesting; soft grippers; modular; benchmarking; robotic manipulation; precision agriculture

Citation: Navas, E.; Fernández, R.;

Armada, M.; Gonzalez-de-Santos, P.

Diaphragm-Type Pneumatic-Driven Soft Grippers for Precision

Harvesting. Agronomy 2021, 11, 1727.

https://doi.org/10.3390/

agronomy11091727

Academic Editor: Baohua Zhang

Received: 5 July 2021

Accepted: 27 August 2021

Published: 29 August 2021

Publisher's Note: MDPI stays neutral with regard to jurisdictional claims in published maps and institutional affiliations.

\section{Introduction}

Although robotics has traditionally been dominated by rigid link designs, during the last two decades this field has undergone a major paradigm shift thanks to the incorporation of soft technologies. The growing interest in the use of new materials has made soft robotics a well-defined research area that aims to improve the interaction between robots and unstructured environments and humans by providing variable adaptability and compliance [1].

In the literature, soft robotics can be found in diverse fields of application, such as medicine and rehabilitation [2,3], assistance [4-6], search and rescue $[7,8]$ and agroforestry [9]. Applications that require manipulation in low-information environments have also been attracting much attention, with prototypes ranging from those attempting to replicate a human hand [10-13] to those that see nature as a source of inspiration for other types of grips [14-17]. Soft grippers can provide significant advantages when there is a need for actuation without precise positioning or without knowledge of the shape or material of the object to be grasped [18]. On the other hand, the ease of manufacture of this type of soft device is remarkable, without the need for heavy machines or machining skills. All these advantages have led many researchers to develop new soft designs, which have been on the rise in recent years and will continue to increase in the coming future.

Another emerging application area where soft robotics can have a significant impact is precision agriculture [19-21] and more specifically, automatic and selective crop harvesting [22,23]. In recent years, the agricultural sector has undergone a deep transformation to cope with the growing demand for food [24-26], introducing advances on machine vision and detection systems [27-30], decision-making architectures [31,32] and autonomous navigation [33]. However, less attention has been paid to improve the dexterous manipulation and the grasping capabilities for selective harvesting. The variability of agri-food products 
and the delicacy with which it is necessary to handle them to avoid bruising make soft robotics especially suitable for this purpose. However, it is important that the proposal for new soft grippers is linked to the fulfillment of certain performance requirements, and therefore, methodologies that enable a comparative evaluation of them should also be envisaged. From an agricultural point of view, these methodologies can include different evaluation criteria, such as the capacity to grasp different types of fruits, grippers size, lifting ratio, power consumption, scalability, response time, surface conditions or technology readiness level (TRL) [34-37]. Otherwise, the literature will tend to be filled with novel but impractical designs.

For this reason, this article aimed to present a new design approach for soft grippers based on modules that combine the use of a pneumatic-driven soft diaphragm actuator and a 3D printed structure. The main advantage of the pneumatic-driven diaphragm actuators over other soft actuators is the ease of manufacture. On the other hand, the 3D printed structure establishes a series of constraints on the degrees of freedom (DoFs) of the soft actuator, which facilitates the control of the gripper. The proposed modules can then be freely configured to obtain grippers adaptable to products of different diameters. Another contribution of this article is to propose a series of easy-to-implement measurement tests to characterize all types of soft diaphragm actuators so that they can serve as the basis for a benchmarking analysis.

The remainder of the article is organized as follows. Section 2 details the steps followed for the design and manufacturing of the modular soft grippers, while Section 3 presents the control system implemented for the pneumatic-driven diaphragm actuators. A proposal for the measurement of the characteristics of soft actuators is described in Section 4 . Section 5 discusses the main results obtained from the experimental evaluation of the proposed soft grippers. Finally, Section 6 summarizes major conclusions.

\section{Design and Manufacturing Approach}

To identify the essential requirements that a soft gripper should meet to be fully operational, agricultural processes and, particularly, harvesting tasks have been reviewed $[19,36,38]$. One of the requirements identified for increasing the profitability of harvesting machinery is the capability to handle different types of crops. For this reason, the goal was to achieve a fully adaptable and modular design concept. The engineered module can be assembled in a variety of ways to obtain a large number of diameters and lengths. This feature allows a gripper designed under this concept to be recombined to adapt it to the harvesting of different types of fruits. Another requirement in this field is simplicity, which translates into interchangeable and easy-to-fix systems. That is why the design approach is based on single modules, which can work independently and are easy to manufacture. Other requirements are more related to the preservation of the quality standards of the fruit than the harvesting process itself, such as avoiding damage to the fruit and the use of non-hazardous materials and designs that prevent the spread of diseases and pests. This important aspect has been ignored in the design of new grippers, whose main disadvantages were the use of materials that could damage the fruits and the complex designs that made cleaning difficult. Hence, the need to use a combination of soft robotics technology with hygienic designs and variable compliance prevents damage to the fruit and the crop. Environmental friendliness, durability and robustness are also important requirements for agricultural and industrial applications. For this reason, the selection of the materials for the manufacturing process has been carefully made to meet these requirements. Finally, the modular soft gripper is designed to be used as the end effector of a robotic manipulator $[39,40]$, being able to perform almost all the harvesting movements required, also known in the literature as picking patterns [20-23]. These picking patterns are in some cases a combination of simple movements, which can be grouped into twisting, pulling, lifting and bending.

In the following, the type of soft material selected and how the material is modeled to achieve the proposed pneumatic-driven soft diaphragm actuators are described. In 
addition, the strengths of the design, which are based on its geometry and modularity, are also explained. Lastly, the manufacture and assembly of the soft grippers modules are presented.

\subsection{Materials}

Several materials, such as Ecoflex [41,42], Dragon Skin [12,43-45] or Elastosil M4601 [42,46-48] are commonly used for soft robotics. Although all of them have been demonstrated to be a valuable option in the manufacturing process due to their ultrasmooth features, it is difficult to know their exact chemical composition since their main applications refer to the manufacture of objects outside the scientific field. Nevertheless, polydimethylsiloxane (PDMS), commercially known as Sylgard 184, has been used in many research applications [41,44,49-52] and allows not only the determination of its mechanical properties, such as high elasticity [53] and thermosetting [49], but also the mathematical modeling of its behavior with great precision by means of a finite element method (FEM) analysis. Another advantage that this material shares with some of the commonly used materials in soft robotics is the ability to cure at room temperature, which is key to simplifying the soft gripper modules' manufacturing process and reducing its cost. However, if required, this process could be accelerated in their industrial manufacture through the use of ovens, which could reduce the curing times from $24 \mathrm{~h}$ to $10 \mathrm{~min}$ at $150{ }^{\circ} \mathrm{C}$ [53]. In addition, PDMS is declared in their safety data sheet as a non-hazardous substance, which guarantees safe interaction with biological products and makes it suitable for agricultural applications. Lastly, PDMS is relatively resistant to fatigue and does not age readily. This means that the PDMS actuator is very suitable for agricultural and industrial applications. Furthermore, PDMS composites that dissipate little energy during normal the operation of cyclic loads (low hysteresis), but dissipate much energy to resist rupture (high toughness), and survive prolonged cyclic loads (fatigue resistant) have recently been proposed [54].

\subsection{Soft Design}

Regarding the geometric design, the grippers proposed in this article consist of singlechannel diaphragm-type actuators. One of the advantages of this kind of geometric design is the simplicity of the process required for its manufacture, which can be divided into two main steps. The first step is the filling of the molds, the diaphragm and the cover, which facilitates molding in comparison to other multiple-channel actuators. The second step is the fixing of these two parts, in which the same material from other parts is used. Another advantage of this kind of soft actuator is the ease of its control. This benefit is because soft diaphragm actuators are designed to only move on one axis, while motion on other axes is considered negligible. Thus, it directly affects the control of the DoFs of the gripper.

The proposed soft diaphragm is also characterized by using the bellows concept, which differs from other geometries in its inflation behavior. In other geometries, such as those based on cylinders, cubes or spheres, the inflation behavior tends not only to produce a forward elongation but also to waste forces on the side walls. This effect tends to reduce the forward advance in both cylindrical and cubic geometries due to radial expansion. A special case is the spherical geometry, which exhibits better inflation behavior than the others, although it has the great disadvantage of requiring intricate molding for its manufacturing process. However, the bellows-based geometry, particularly the proposed bellows-cylinder geometry, can be studied as a combination of cylindrical and spherical shapes. This approach partially solves the problem described because the forces applied by radial inflation are also used for forward elongation, extending almost the entire body of the gripper in that direction. The difference between these geometries can be observed in Figure 1. 


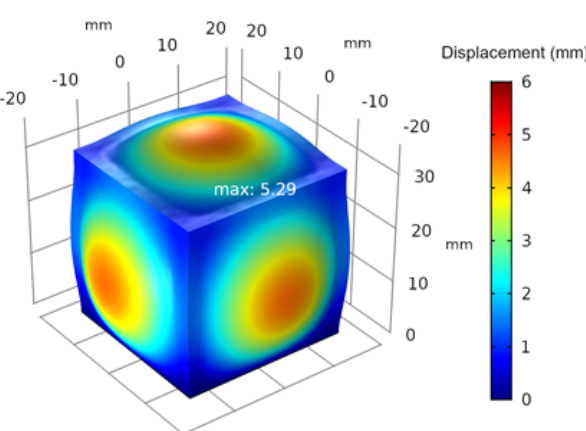

(a)

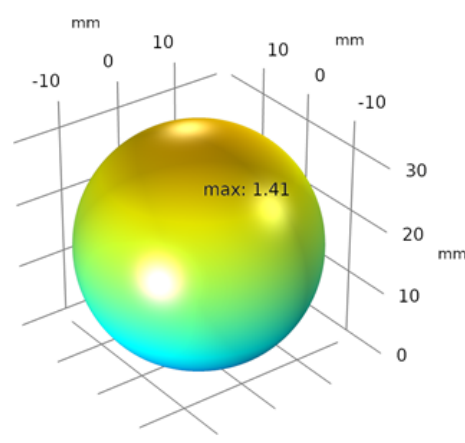

(c)

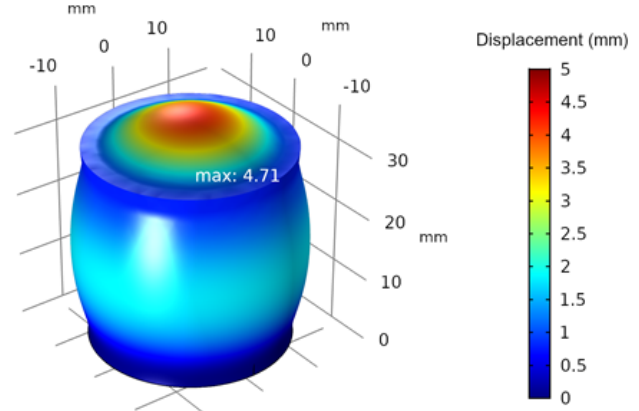

(b)

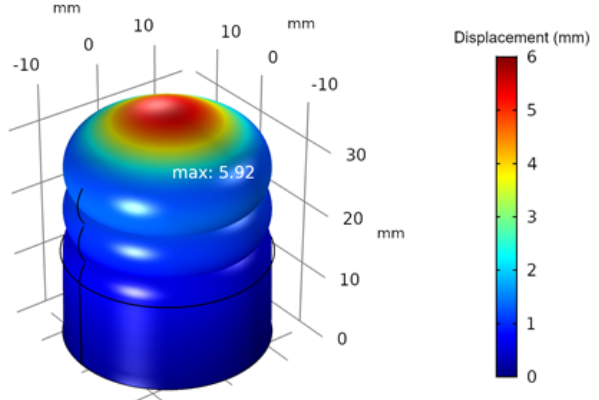

(d)

Figure 1. An example of the inflation behavior of $30 \mathrm{~mm}$ geometries with $3 \mathrm{~mm}$ walls under an internal pressure of $50 \mathrm{kPa}$. (a) Cubic geometry. (b) Cylindrical geometry. (c) Spherical geometry. (d) Bellows-based geometry. As can be observed in (a) and (b), part of the air flow is wasted on the expansion of the walls to different degrees depending on the geometries, which does not contribute to the forward displacement. This method does not seriously affect sphere-based geometry, but due to manufacturing criteria, bellows-based geometry is an important option to consider.

With the purpose of designing suitable soft grippers for medium- to large-sized fruits, two types of soft diaphragms are considered: one with smaller bellows than the other but both with the same diameter. To determine their inflation behavior, the two designs were modeled in COMSOL Multiphysics ${ }^{\circledR}$ using an FEM (see Figure 2). For this, PDMS was modeled as a hyperelastic material. In the literature, several mathematical models can be found to describe the behavior of this type of soft material. However, the secondorder Ogden model, compared with Mooney-Rivlin's or Neo-Hookean's models, more accurately represents its response [55]. Furthermore, due to the inflation pressure, the soft diaphragm will be under equibiaxial tension. This type of tension in hyperelastic materials, which is the case for the PDMS material, was theorized by Ogden [56]. This method involves elastic solids with a strain-energy function and isotropic behavior relative to the stress-free ground state. It also assumes that the solid is incompressible. Thus, it can be formulated as follows:

$$
\sigma_{i}=\mu_{r} a_{i}^{\alpha_{r}}-p,
$$

where $\sigma_{i}, i \in\{1,2,3\}$, represents the principal Cauchy stresses $\left(\sigma_{1}, \sigma_{2}, \sigma_{3}\right)$, the parameters $\mu_{r}$ and $\alpha_{r}$ are experimentally obtained constants, $a_{i}$ represents the stretches $\left(a_{1}, a_{2}, a_{3}\right)$ and $p$ is an arbitrary hydrostatic pressure introduced because of the incompressibility constraint. Due to the equibiaxial tension, two out of three principal stresses are equal, and the third one is zero: 


$$
\sigma_{2}=\sigma_{3}=\sigma, \sigma_{1}=0
$$

Moreover, the stretches can be written as follows:

$$
a_{2}=a_{3}=a,
$$

and due to the incompressibility assumption, it can be considered that $a_{1}=a^{-2}$. The substitution of the aforementioned into (1) is as follows:

$$
\sigma_{i}=\mu_{r} a^{\alpha_{r}}-p, 0=\mu_{r} a^{\alpha_{r}-2}-p .
$$

The elimination of $p$ yields:

$$
\sigma_{i}=\mu_{r}\left(a^{\alpha_{r}}-a^{\alpha_{r}-2}\right) .
$$

Finally, Equation (5) is inserted into the FEM software together with the values of $\mu, \alpha$ and bulk modulus, which were obtained from [55]. The PDMS mix ratio used was a 15-part base elastomer and a one-part curing agent. The data obtained from the FEM software were shown in Figure 2 for the soft actuators, and in Figure 3 for a gripper with a hexagonal configuration. All cases were analyzed under an air pressure of $50 \mathrm{kPa}$.

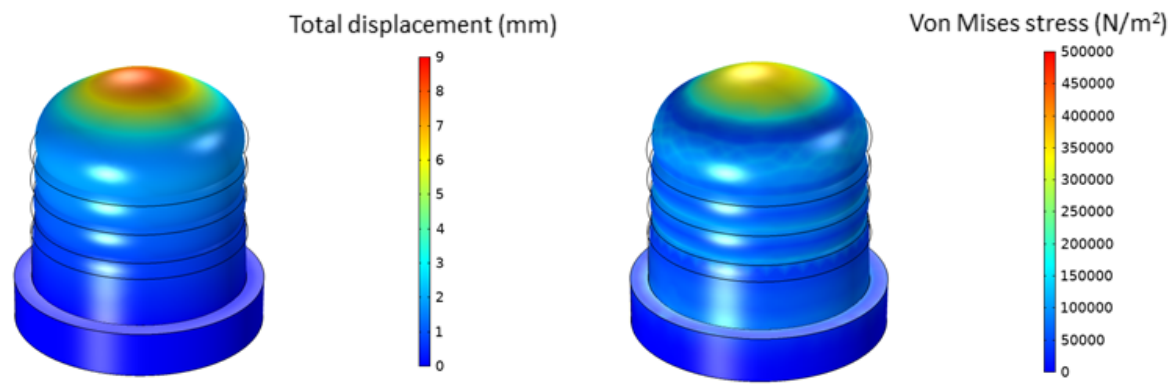

(a)

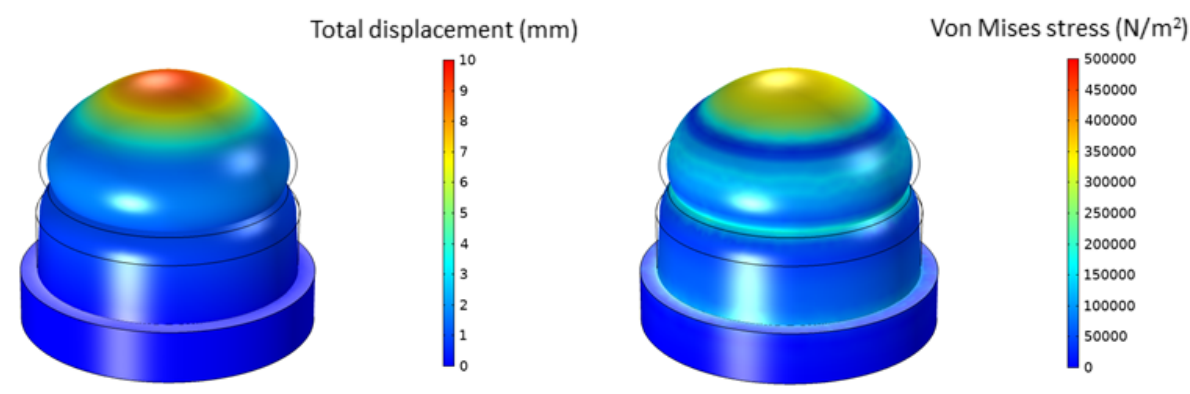

(b)

Figure 2. Model showing the displacements reached and the von Mises stress under $50 \mathrm{kPa}$ pressure: (a) 4-bellows soft actuator; and (b) 2-bellows soft actuator. 


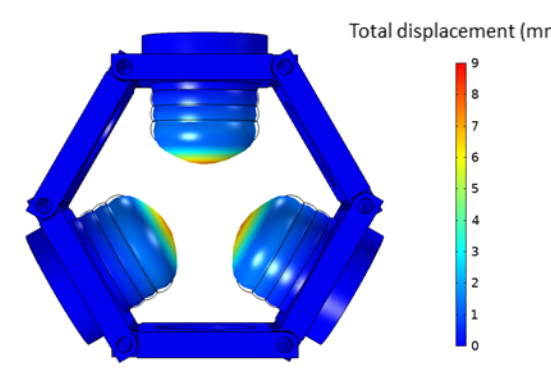

(a)

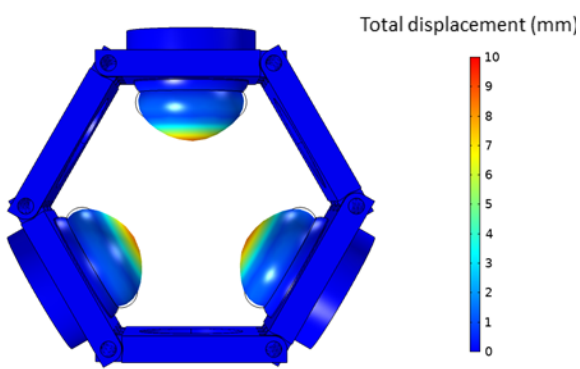

(b)

Figure 3. Model showing the displacements reached on the actuators of the soft gripper with hexagonal configuration under constant pressure: (a) 4-bellows soft actuator; (b) 2-bellows soft actuator.

As can be seen in the figures above, the FEM analysis yields a larger displacement for the 2-bellows actuator than for the 4-bellows actuator-10 and $9 \mathrm{~mm}$, respectively. Regarding the stresses, both actuators have a tensile strength of approximately $6.7 \mathrm{MPa}$, which is the value indicated by the manufacturer.

\subsection{Design of the Rigid Structure}

One of the largest challenges in agricultural automation is to design a gripper that can be multipurpose, i.e., it can harvest different types of fruits with minimal modifications. Referring to soft robots, and more particularly to soft grippers, the aforementioned task becomes even more intricate because this type of robotics requires a prior design of the gripper, which obviously limits its scope. In the literature, a few research studies that explore the concepts of modularity and scalability can be found. For example, the soft gripper described in [49] presents a modular design, but it is only conceived for small fruits and edible fungi, with simplified computational modeling and relatively minor displacement, at the same inlet pressure, than the proposed soft actuator.

This article aimed to go one step further and advance in modularity and scalability to achieve a quasi-universal gripper concept adaptable to each fruit. To this end, a 3D-printed module is designed and made of polylactic acid (PLA), which is one of the best-known synthetic biodegradable polymers, with good mechanical strength and low toxicity. Its faster degradation makes it an environmentally friendly option compared to traditional plastics [57]. As higher toughness is desired for durable applications, research progress on toughening PLA based on plasticization, copolymerization, and melt blending with different tough polymers, rubbers and thermoplastic elastomers can be found in several recent review articles [58-64]. In addition, many PLA formulations with improved toughness are available in the market for durable applications, as summarized in [65]. The proposed PLA structure has the following advantages. First, the module is independent of the gripper; i.e., it can work on its own and make the gripper adaptable to a wide variety of tasks. Second, the gripper is interchangeable, which makes it deeply reliable. This novel feature is an advantage over other soft grippers, which are usually formed in a single body configuration. Therefore, if any failure occurs in these devices, it is necessary to change the entire gripper. Third, this module is completely replicable: as both the rigid components and the mold of the soft components can be easily 3D printed, the materials used for their manufacture are fully available and inexpensive, and there is no need for the postprocessing of the parts. The main parts of the soft gripper module can be seen in Figure 4 . 


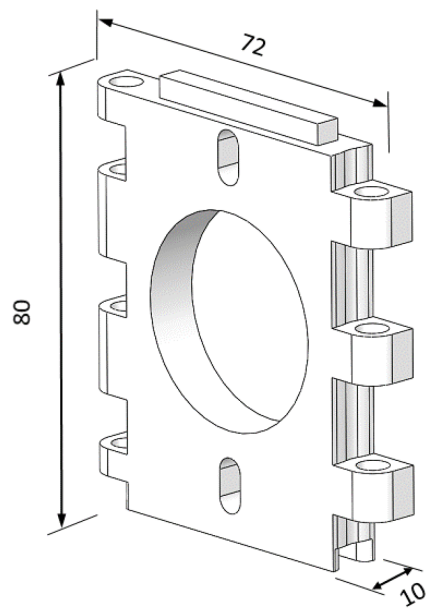

(a)

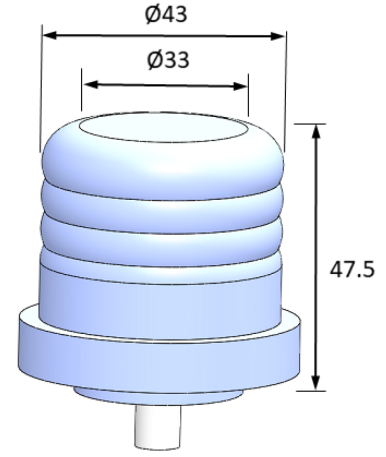

(b)

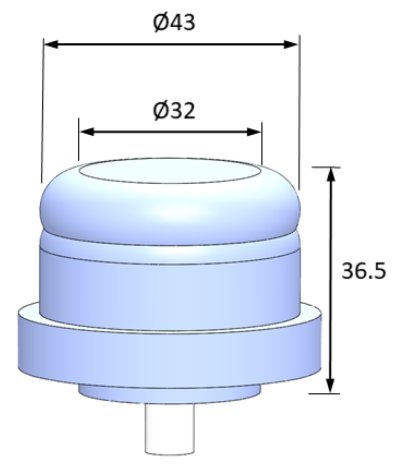

(c)

Figure 4. Main parts of a single soft gripper module with the two types of actuators: (a) rigid structure; (b) 4-bellows actuator; and (c) 2-bellows actuators. All dimensions are in mm.

Finally, the proposed design concept allows the soft grippers to be configured with diverse geometries, which makes them fully adaptable not only to different types of fruits and vegetables but also to different manipulation methods. Figure 5 shows several examples of soft grippers obtained from different configurations of the proposed modules.

In Figure 5a, several closed gripper configurations are presented. These close configurations are suitable when multiple points of contact are needed. Thus, these grippers offer better control of the object and are capable of performing almost all the required harvesting movements or picking patterns. On the other hand, the proposed modules can also be configured in open configuration, as shown in Figure 5b. Unlike close chain configurations, which are intended to harvest fruits that are always hanging vertically, open chain configurations are indicated for medium-to-large sized fruits that usually lay on the ground, such as watermelons, melons or pumpkins.

It is also worth mentioning that the interaction between the soft components and the rigid structure was studied by FEM, confirming that no relevant load values are presented in the structure. 

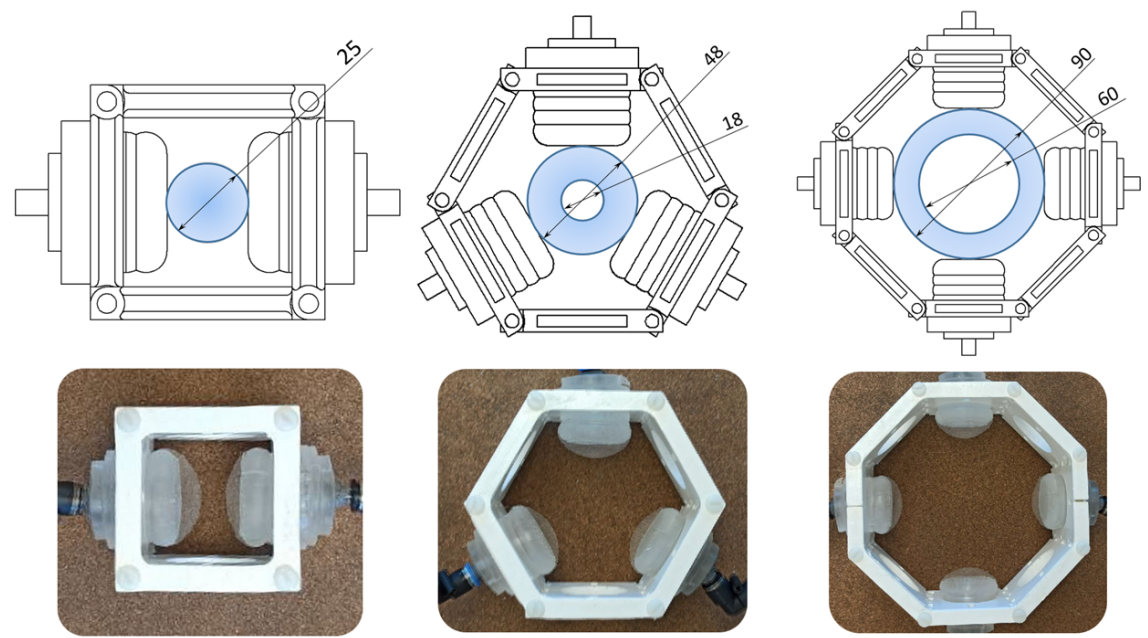

(a)
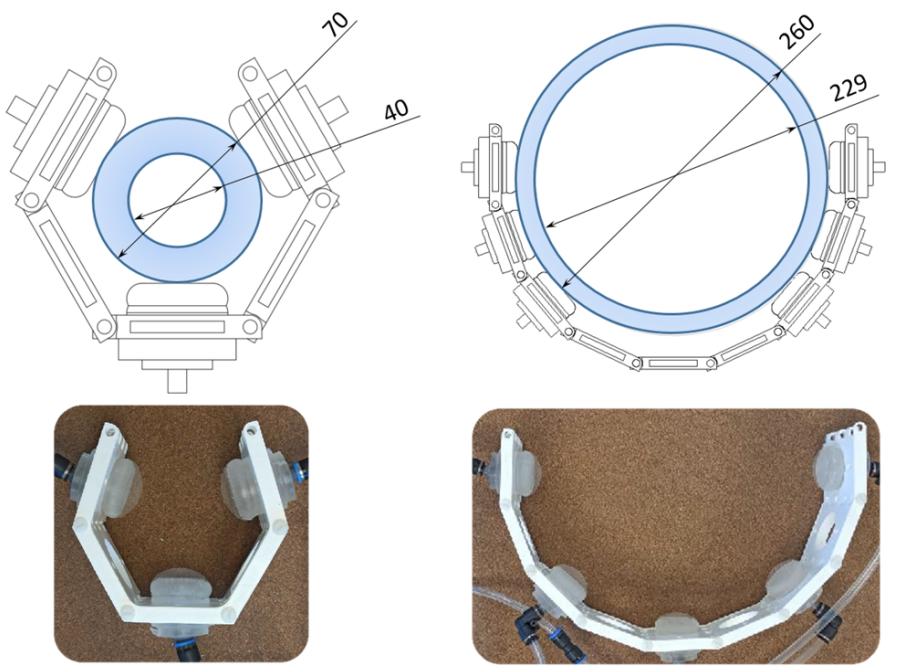

(b)

Figure 5. Examples of soft grippers obtained from the proposed design approach: (a) closed gripper configurations; and (b) open gripper configurations.

\subsection{Manufacturing and Assembly}

For the manufacture of the gripper actuators, a molding process widely used and discussed in the soft robotics literature was followed [66-68]. A graphical summary of the molding process is shown in Figure 6.

The process is described as follows: (i) The mold is printed by a 3D printer; in this case, the plastic used was PLA; (ii) once the mold is mounted (Figure 6a), PDMS is poured over it (Figure 6b); (iii) then, the two molds with PDMS are placed in a vacuum chamber (Figure 6c) to eliminate internal bubbles. After this vacuum process, the entire assembly is left to cure at room temperature for one to two days, depending on the environmental conditions; (iv) next, the demolding process is carried out (Figure $6 \mathrm{~d}$ ), gluing the two resulting parts with PDMS (Figure 6e); and (v) finally, after waiting one day, the soft actuator is fully operational (Figure 6f). In some cases, a silicone sealant, Loctite 5699, is applied to prevent air leakage between the polyurethane (PUR) pipe and the PDMS of the soft actuator. 


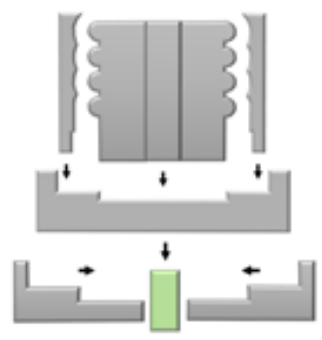

(a)

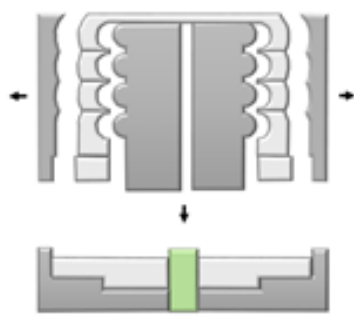

(d)

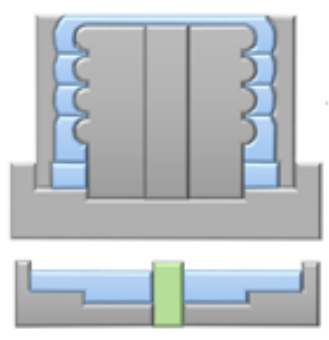

(b)

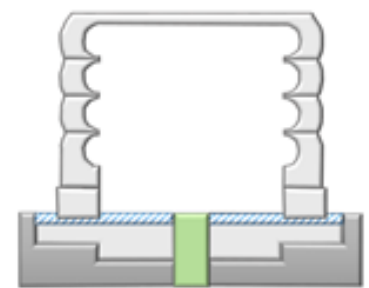

(e)

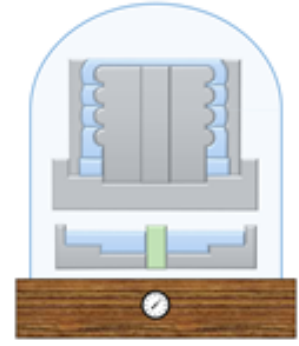

(c)

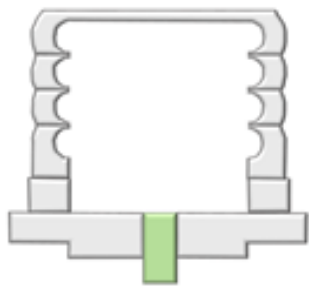

(f)

Figure 6. Molding process. The PLA mold is shown in dark gray, the fresh PDMS soft gripper is represented in blue, the precured PDMS is in striped blue, the cured PDMS soft actuator is displayed in light gray and the PUR pipe is in green. (a) Mold assembly. (b) Pouring of the PDMS on the mold. (c) Vacuum process. (d) Demolding. (e) Gluing with PDMS of the two resulting parts. (f) Fully assembled soft actuator.

Although the ease of manufacture of the proposed approach is remarkable, it is worth mentioning the need for repeatability and accuracy studies that research how to mitigate the common effects that appear in soft actuators, such as delamination or interstitial bubbles that can be the result of faulty manufacturing. To solve these problems, several solutions have been proposed, such as the use of vacuum chambers [47,69-71], for which positive results have been reported. However, it is impossible to find in the literature a method where, for example, variables such as pressure or time are controlled as a function of volume to ensure the repeatability of the process. Procedures based on the 3D printing of soft materials, as well as lost wax manufacturing, may become interesting options in the future, given their greater possibilities for achieving repeatability and accuracy during the manufacturing process.

Once the soft actuator is manufactured and the rigid components are printed with a $10 \%$ infill, the soft part is inserted into the hole of the rigid structure and attached to it via a screwed clip. With all this, the standalone module is fully assembled. To mount the modules for different gripper configurations, nylon threaded rods, washers and nuts can be used (see Figure 7a). The preference for the use of nylon fasteners is due to their low-density property, which is useful for lightweight robotic manipulators. However, it can be replaced by steel if the application requires it. A fully assembled module and some examples of soft grippers' configurations are shown in Figure $7 \mathrm{~b}$.

One of the main advantages of the proposed design approach is that it allows the rigid structures of the modules to be assembled following various geometries that facilitate the positioning of the soft actuators to ensure sufficient contact areas that provide stable grips for different types of fruits. Figure 8 shows that due to the proposed design, even other grips, different from the ideal one (i.e., one in which the fruit is centered at the midpoint of the gripper) are equally feasible. If the object (represented by the blue areas in Figure 8) and the closed configuration gripper are concentric, all the actuators will be involved in the grasping. On the contrary, if the target is located in other areas, such as in the orange areas, the grasping could be carried out by means of only two actuators. 


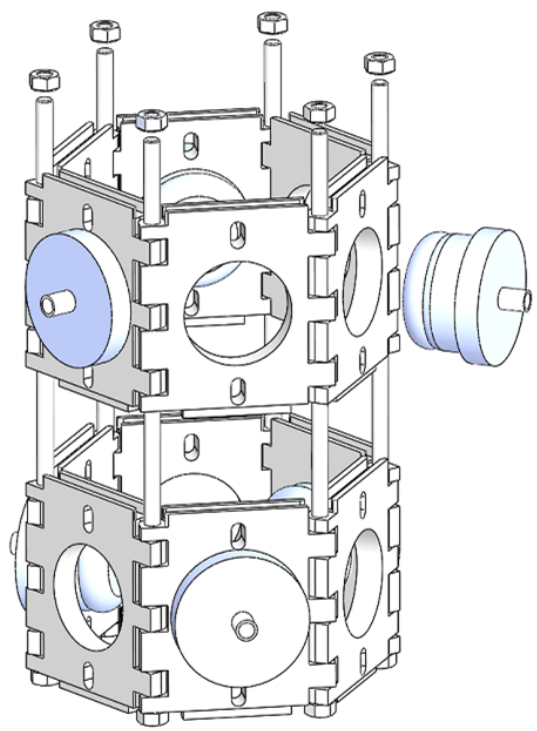

(a)

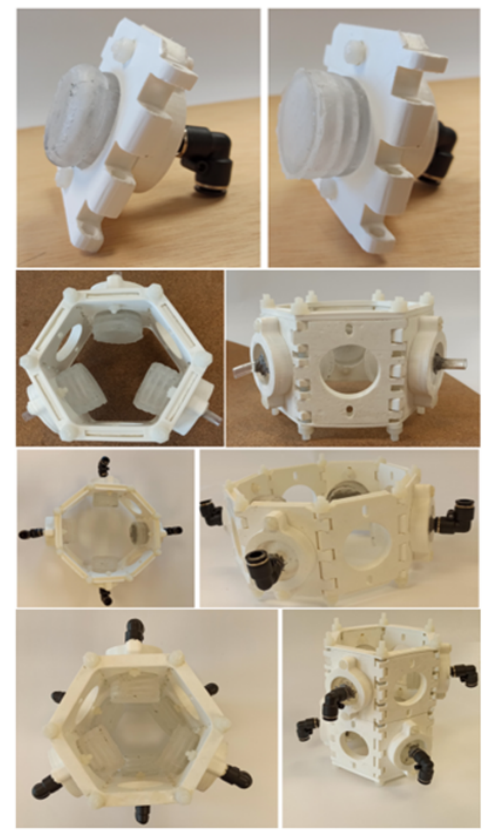

(b)

Figure 7. Modules, assembly process and various configurations. (a) Assembly of the modules. (b) Fully assembled modules and several soft grippers configurations.
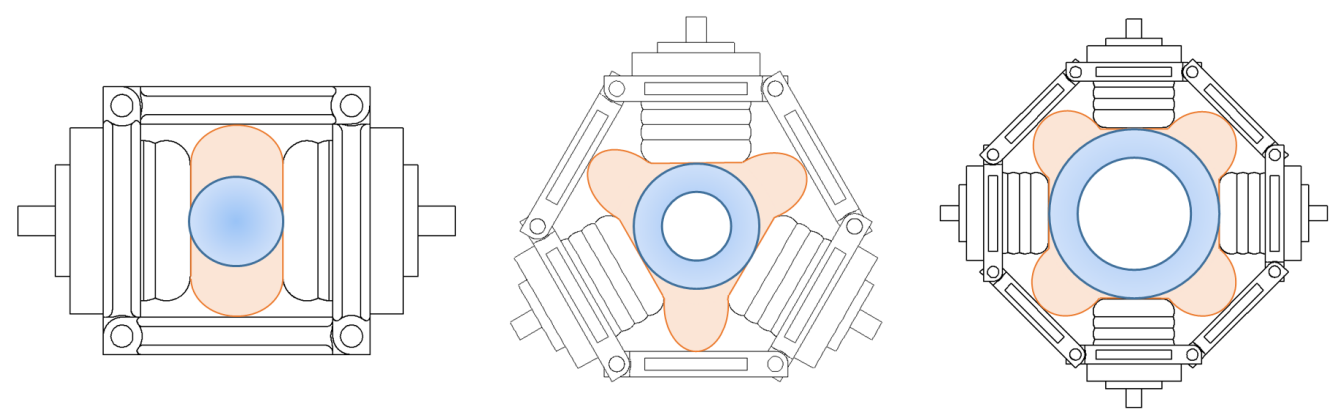

(a)
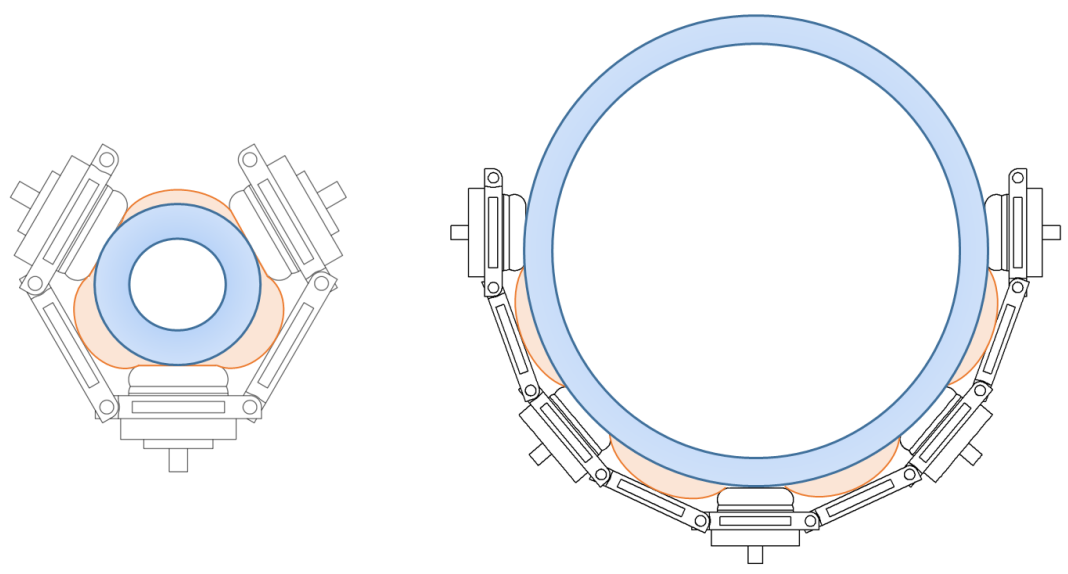

(b)

Figure 8. Feasible grip areas. (a) Closed gripper configurations. (b) Open gripper configurations.

As can be seen in Figure 8a, the closed configurations offer a larger grasping area than open configurations, as shown in Figure $8 \mathrm{~b}$, since the object can even be placed between the rigid part, which acts as a fulcrum, and the soft actuators. Furthermore, it could be 
derived that the grasping points of an object in closed configurations will be from 2 to $n$, where $n$ is the number of soft actuators placed on the gripper, which depends on the fruit. On the other hand, in open configuration, it is necessary to adapt the pressure of each actuator independently, since generally the actuators at the ends support the load of the target, while those on the inside help to prevent its rotation during the grasping. Another important advantage of the proposed design approach is that the 3D printed structure establishes a series of constraints on the DOFs of the soft actuator, which facilitates the control of the grippers.

\section{Control System}

Deformability and compliance are some of the main characteristics of soft robotics, which translate into a large intrinsic number of DoFs. This obviously affects the control system in terms of complexity. However, such DoFs, given the elastic behavior of the material, offer the possibility of performing movements such as bending, twisting, stretching, compression, and buckling wrinkles [72]. Typically, the soft control barrier is compensated by a high level of sensorization of the systems. Other authors [73] have used real-time FEMs to control soft elastomer robots. This approach can be a good option, as long as a good mathematical model of the material is used, which is complicated for certain soft materials.

On the other hand, authors such as [74] used rigid components embedded in soft actuators. This hybrid concept was developed in recent decades as a combination of soft robotics and rigid robotics $[74,75]$. The gripper concept proposed in this article can be studied as a particular case of a hybrid grippers, not only because there is a physical combination between these two technologies but also because the rigid structure establishes a series of constraints on the DoFs of the soft actuator, which facilitates the control of the gripper. The modular soft grippers are then controlled by a LabView interface, from which the pneumatic electrovalves can be activated and their pressure can be manually adjusted.

Furthermore, as can be seen in Figure 9, the soft actuators can operate automatically via proportional-integral-derivative (PID) control, where the feedback is the internal pressure measurement provided by the pressure sensor located at the inlet of the soft actuator. This controller reads the pressure change with a frequency of $50 \mathrm{~Hz}$, and if its rate of change is greater than that caused by the electropneumatic regulator, it means that the soft actuator is in contact with the object due to the decrease in the volume of the gripper and the increase in the actuator internal pressure (see the red point in Figure 9). This makes it possible to monitor the contact of the soft actuator without sensors embedded in it.

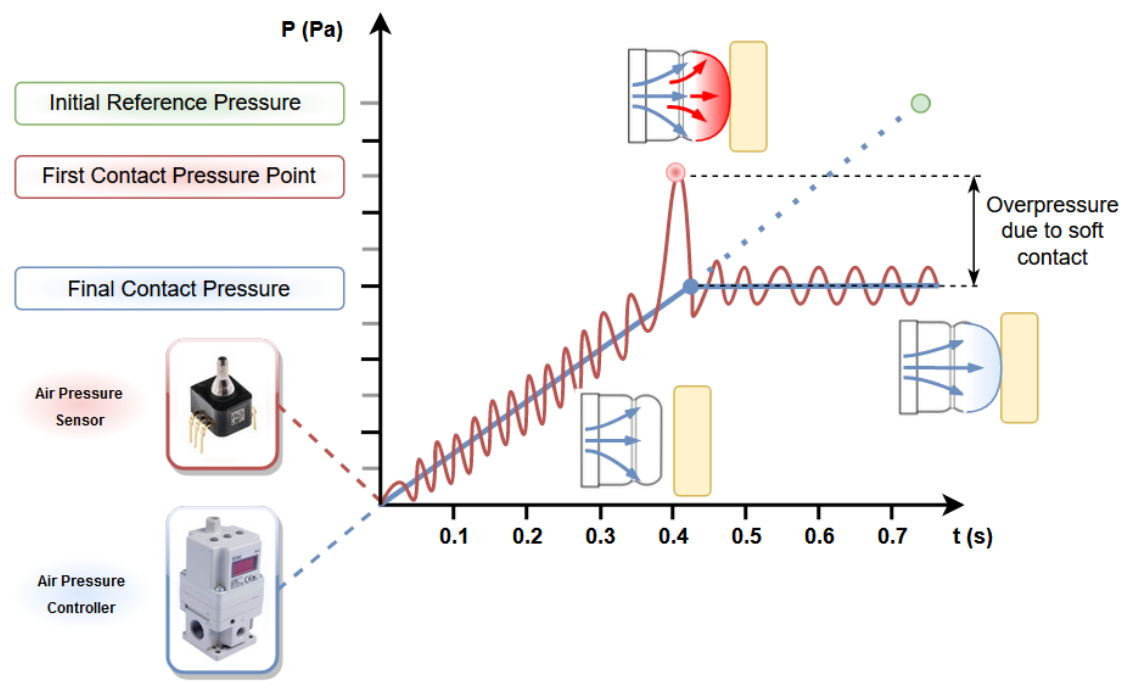

Figure 9. Diagram of the control method for the soft actuator without embedded sensors. 


\section{Electronic and Pneumatic System}

To drive the modular soft grippers, sensor and control elements are required to ensure the accuracy of the air pressure measurement and a constant airflow. In Figure 10, the electronic and pneumatic systems are schematically described.

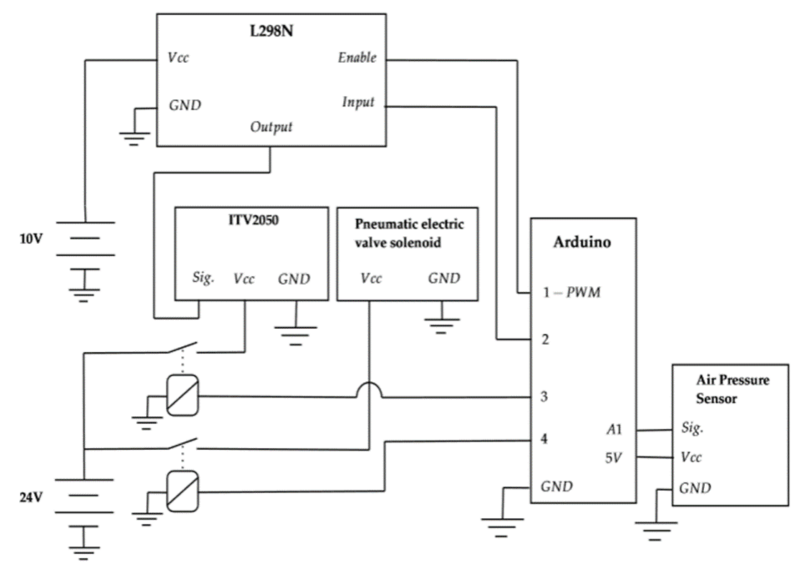

(a)

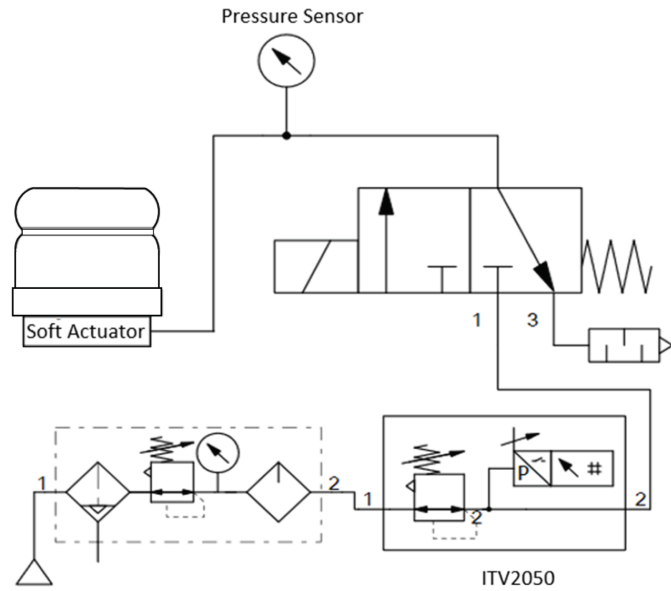

(b)

Figure 10. Electric and pneumatic systems. (a) Electric scheme of the modular soft grippers. (b) Pneumatic scheme.

Figure 10a shows that the core of the electronic system is an Arduino, particularly a Mega 2560, which controls the different elements by means of a LabVIEW interface. Table 1 shows the features of the air pressure regulator used. Furthermore, Figure 10b displays the pneumatic system, which consists of (i) an Abart Start O15 air compressor with a power of six liters and $1.1 \mathrm{KW}$; (ii) pneumatic air treatment equipment; (iii) a pneumatic solenoid valve; (iv) an SMC ITV2050 electropneumatic regulator; and (v) a Honeywell 40PC air pressure sensor (measurement range: $0-100 \mathrm{kPa}$, output voltage: $0.5-4.5 \mathrm{~V}$, measurement precision: $\pm 0.4 \%)$. 
Table 1. Electropneumatic Regulator ITV2050 specifications.

\begin{tabular}{cc}
\hline Characteristic & Value \\
\hline Pressure range & $0.005-0.9 \mathrm{Mpa}$ \\
Max. supply pressure & $1.0 \mathrm{MPa}$ \\
Power consumption & $4 \mathrm{~W}$ \\
Max. flow rate & $1500 \mathrm{~L} / \mathrm{min}$ \\
Input signal & $0-10 \mathrm{Vdc}$ \\
Mass & $0.350 \mathrm{~kg}$ \\
Linearity & $\pm 1 \%$ \\
Hysteresis & $0.5 \%$ \\
Repeatability & $\pm 0.5 \%$ \\
Response time & $0.1 \mathrm{~s}$ \\
\hline
\end{tabular}

\section{Determination of Soft Actuators Features}

One of the main gaps that has not yet been addressed in soft actuators is the definition of a method to determine their characteristics. However, it is clear that there is a need for a reliable method that can quantify the soft actuator features to facilitate a comparative study between the different models and establish not only different categories according to their capabilities but also an index of improvement in the soft technology.

One of the most important characteristics of a soft gripper is the contact force that it can exert on an object and its control, since its ability to grasp more or less sensitive objects depends on this. The research studies presented in $[12,48,49]$ use different types of contact force measurements. In [49], the contact pressure $\left(P_{c}\right)$ is found by means of FEM software, and the contact area $\left(A_{c}\right)$ is measured by the "fingerprint" of the soft gripper on a Styrofoam surface. The contact force $\left(F_{\mathcal{c}}\right)$ is found by the well-known Equation:

$$
F_{c}=P_{c} \cdot A_{c}
$$

Although it is a nice approximation, the method used to find the contact pressure is not very reliable because it depends on the mathematical model implemented in the FEM software, the Mooney-Rivling model, which describes the behavior of PDMS with low accuracy [55].

In [12], the measurement process is divided into three tests. In two of them, the procedure is a trial and error, whereas in the last one, the Takei Physical Fitness Test is performed. However, this test is only suitable for humanoid hands, so it is not applicable for diaphragm-type grippers. Finally, another interesting measurement process can be found in [48], where a pressure map is used. In the latter case, the pressure map is wrapped in a tube with a radius adjusted to an appropriate curve for the soft gripper.

Thus, there is no standardized tests for measuring soft gripper properties. For this reason, this article aims to propose a measurement process using two measuring devices by which soft grippers can be objectively analyzed, particularly those of the soft diaphragm type.

Figure 11 shows the two devices used in the proposed processes to measure the various features of soft actuators, which are the relation between the forward displacement, contact force, contact area, contact pressure in the middle of the soft actuator and the inlet air pressure.

The first parameter, the forward displacement, is measured in a press by means of a dial gauge with an accuracy of $0.01 \mathrm{~mm}$, the scheme of which can be seen in Figure 11a. The other parameters, which are the contact force, the contact area and the contact pressure, are measured using a press, such as the one outlined in Figure 11b, with an Entran ELWD1-500N compression load cell, whose technical specifications are listed in Table 2. This load cell is selected because its load reading range is within the expected values for this type of pneumatic actuator. 
Table 2. Main technical specifications of the compression load cells.

\begin{tabular}{cc}
\hline Characteristic & Value \\
\hline Nonlinearity & $\pm 1 \%$ \\
Hysteresis & $\pm 1 \%$ \\
Thermal Zero Shift & $\pm 2.5 \mathrm{mV} / 50{ }^{\circ} \mathrm{C}$ \\
Thermal Sensitivity & $\pm 2.5 \% / 50{ }^{\circ} \mathrm{C}$ \\
Deflection at "FS" & $<0.013 \mathrm{~mm} \mathrm{nom.}$ \\
Operating Temperature & $(-40 \text { to } 120)^{\circ} \mathrm{C}$ \\
Thickness & $3.81 \mathrm{~mm}$ \\
Diameter & $25.4 \mathrm{~mm}$ \\
\hline
\end{tabular}

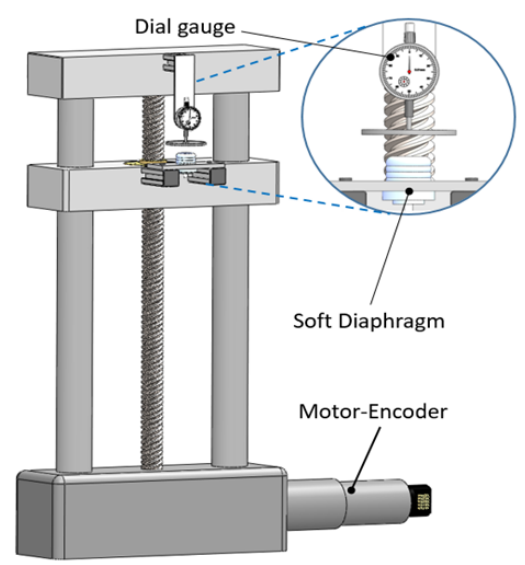

(a)

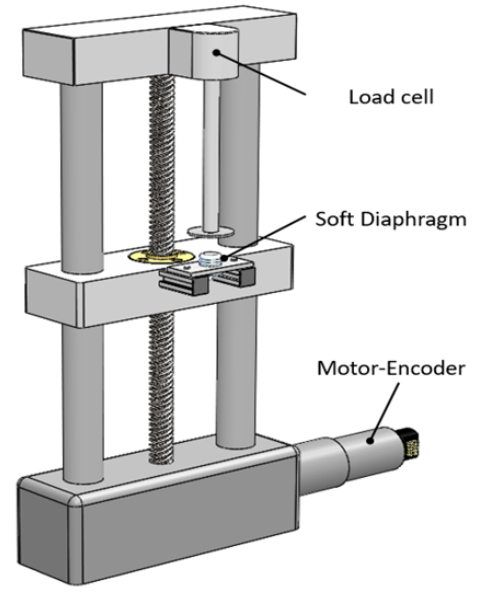

(b)

Figure 11. Devices for measuring the soft actuators features. (a) Assembly of the dial gauge to measure the displacements in the soft diaphragm. (b) Assembly of the load cell to measure the contact force in the soft diaphragm.

To proceed with the measurement process, it is necessary to take into account some basic knowledge of geometry, which is better explained with the graphic description shown in Figure 12.

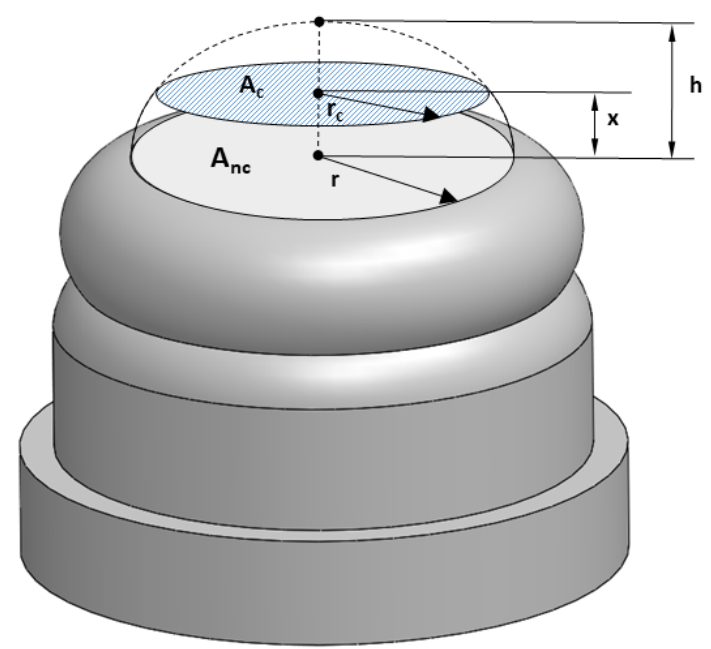

Figure 12. Graphic description of the contact area and the non-contact area, where $r$ is the actual radius of the soft actuator, $r_{c}$ is the radius of the contact area, $x$ is the distance between the actuator and the object and $h$ is the relative longitudinal distance between the soft actuator at rest and its maximum displacement at a given pressure. 
Therefore, taking into account the diverse cases, the contact area can be stated as follows:

$$
A_{\mathcal{c}}(x)=\left\{\begin{array}{lr}
\pi r^{2}, & \mathrm{x}=0 \\
0, & x>0 ; h<x \\
\pi r_{c}^{2}, & x>0 ; h>x
\end{array}\right.
$$

where $r_{c}$ can be expressed through various geometric relationships as: $r^{2}-(x+r-h)^{2}$.

Therefore, the contact area will be accurate for each pressure and displacement. The only assumption made was that the contact area was circular, which has been experimentally determined to be valid for this actuator geometry.

Once the contact area was obtained and the contact force was measured, the contact pressure in the middle of the soft actuator can be obtained by the contact pressure distribution formulated by [76], here presented in Equation (8):

$$
p\left(r_{v}\right)=C_{k} \frac{N}{\pi r_{c}^{2}}\left[1-\left(\frac{r_{v}}{r_{c}}\right)^{k}\right]^{\frac{1}{k}}
$$

where $N$ is the normal force, $r_{c}$ is the radius of the contact explained above, $r_{v}$ is the variable radius, $0 \leq r_{v} \leq r_{c}, k$ determines the shape of the pressure profile, and $C_{k}$ is a coefficient that adjusts for the profile of the pressure distribution over the contact area to satisfy the equilibrium condition.

In [77], the author shows that for soft contact, the value of $k$ is approximately 1.8. With that, the value of $C_{k}$ can be calculated as follows:

$$
C_{k}=\frac{3}{2} \frac{k \Gamma\left(\frac{3}{k}\right)}{\Gamma\left(\frac{1}{k}\right) \Gamma\left(\frac{2}{k}\right)}
$$

Since $p_{0}$, which is the pressure in the middle of the actuator, can be a target value for evaluating a soft actuator, $r_{v}$ is substituted by 0 to obtain the contact pressure in the middle of the soft actuator, leaving Equation (8) as

$$
p_{0}=p(0)=C_{k} \frac{N}{\pi r_{c}^{2}}
$$

With all of the above, the proposed measurement process has been described in order to characterize the soft actuators in terms of forward displacement, contact force, contact area and contact pressure.

\section{Evaluation}

In order to validate the feasibility of the proposed approach and evaluate its performance, several experimental tests were carried out.

First, two types of soft actuators were characterized following the method described in Section 4, one with four small bellows and the other with two larger bellows, with the aim of comparing their behavior. The graphs in Figure 13 show the experimental results obtained during these tests.

As can be observed in Figure 13a, the two soft actuators exhibit an almost linear behavior and a similar performance to that obtained in the FEM simulations. In addition, it has been experimentally proven that the 2-bellow actuators can reach more force than the 4-bellow actuators, as shown in Figure 13b. Thus, it can be concluded that the thicker the actuator walls are, the more force they can exert due to the input pressure that this actuator can handle. However, if the walls are thinner, then the reliability of the actuator is reduced, even though the displacements may increase. Therefore, the wall thickness should be a variable in the actuator design. This thickness might be different depending on the parts of the actuator in order to take advantage of the chosen geometry. Figure $13 \mathrm{c}$ shows how the area increases almost linearly when the object is separated from the actuator 
by a certain distance. This behavior is because the contact surface is flat, with an area larger than the actuator head. In Figure 13d, the results of the two previous graphs are displayed, where it can be observed that the contact pressure will remain constant due to the conditions of the experiment. During the tests, several behaviors are also observed among actuators of the same type. This finding is due to the manufacturing process, which causes the existence of some bubbles in the actuators. Finally, it can also be deduced from the different experiments carried out that for the same actuator bellows diameter, short bellows tend to behave as cylindrical geometries, which shortens their displacement, while high bellows improve their displacement due to their spherical-like behavior.
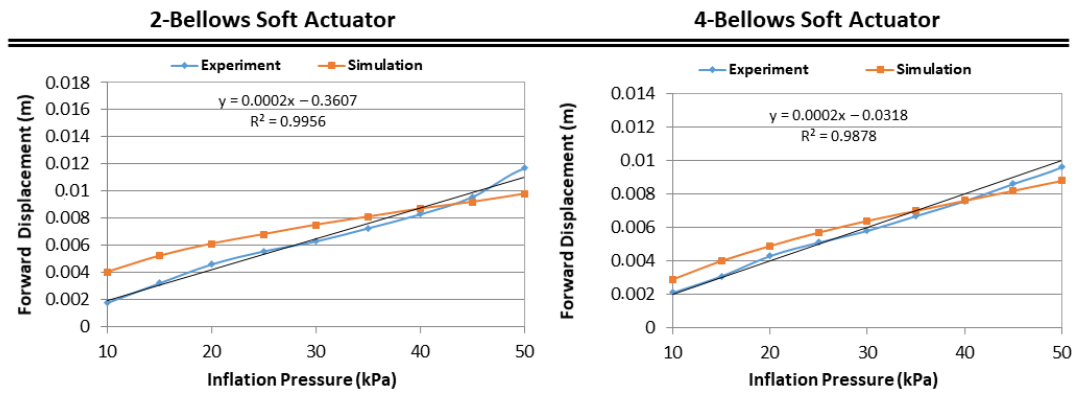

(a)
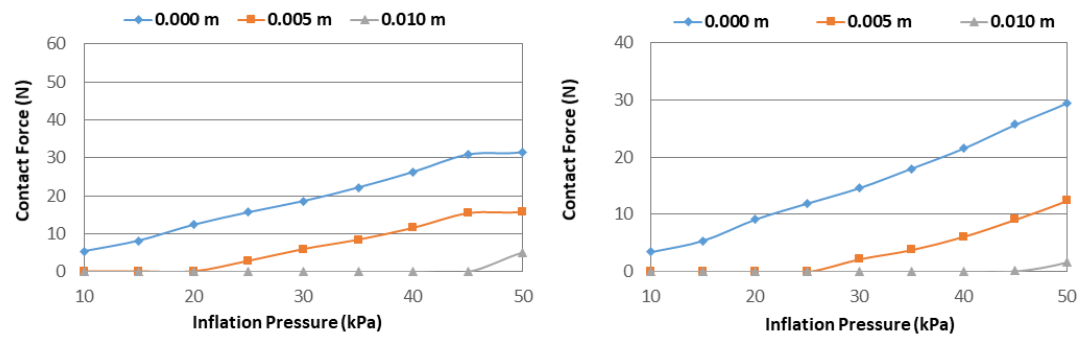

(b)
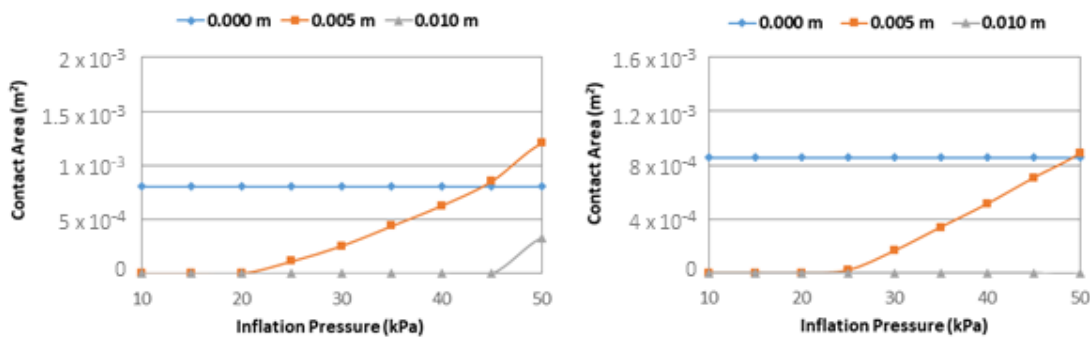

(c)

Figure 13. Cont. 

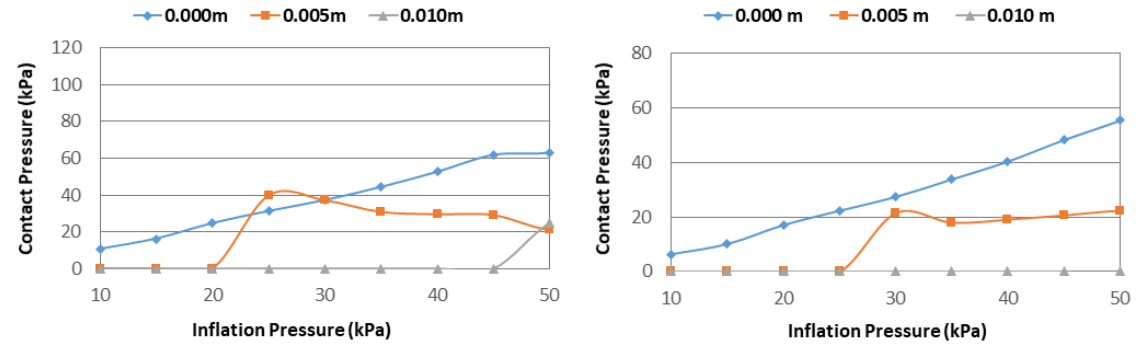

(d)

Figure 13. Experimental characterization of soft actuators. (a) Experimental measurement of the forward displacement as a function of the inflation pressure. (b) Measurement of the contact force as a function of the inflation pressure. (c) Relationship between the contact area and the inflation pressure. (d) Relationship between the contact pressure in the middle of the soft actuator and the inflation pressure. Graphic legends show the distance between the soft actuator and the object.

Then, to evaluate the gripping force of the modular soft gripper, a grasping force test [68] was conducted. This test, also known in the literature as a slip payload test [49] or pull-off force test [67], was performed by using a system designed to both generate a downward force and measure the slip payload. The system setup can be seen in Figure 14 .
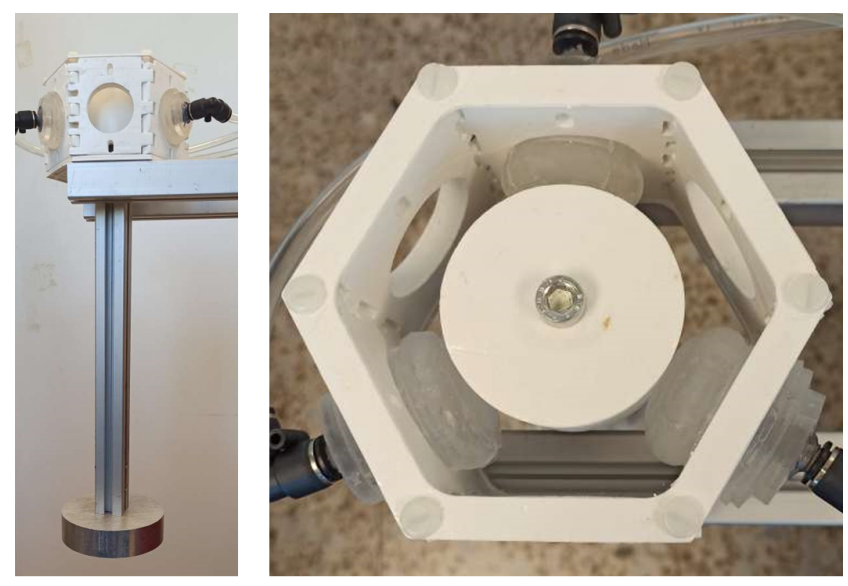

(a)
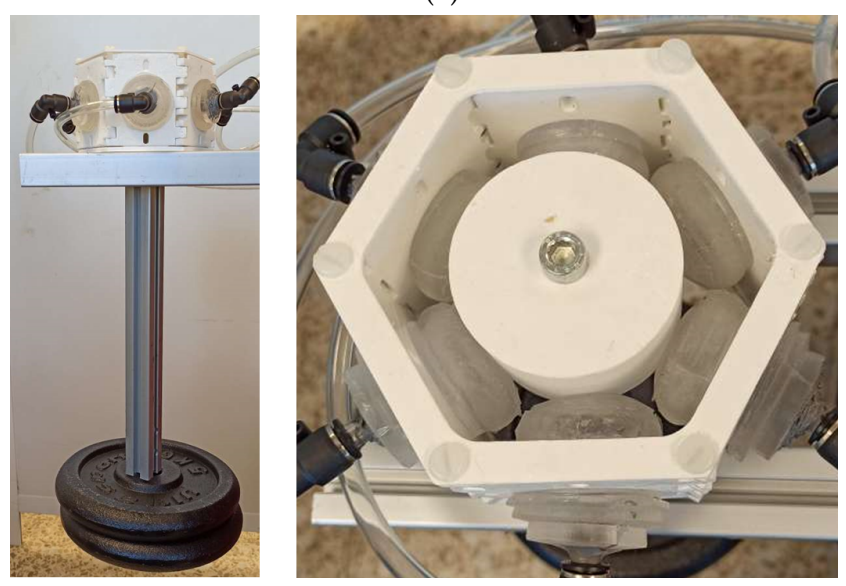

(b)

Figure 14. Slip test setup in a hexagonal configuration: (a) Soft gripper with three soft actuators; (b) Soft gripper with six soft actuators. 
In this slip payload test, a constant pressure of $50 \mathrm{kPa}$ is used, and load is added until either slippage occurs due to the vertical force exceeding the frictional forces of the gripper, or until the failure or cracking of the soft actuator occurs. The test result is a limit load of $4.75 \mathrm{~kg}$ and $10 \mathrm{~kg}$ for a hexagonal configuration of three and six soft actuators, respectively. This limit load is sufficient to grasp most of the fruits on the market and does not limit the load that a normal robotic manipulator can lift. This test also shows the modularity and scalability of the proposed approach, considerably increasing the load capacity of the gripper, without significantly influencing its structural design.

Several tests were also carried out to evaluate the grasping performance, as well as the possible damages produced in real harvested fruits. To that end, a hexagonal configuration gripper endowed with three soft actuators was mounted on one of the Kinova Mico manipulators [78] that make up the ROBOCROP dual-arm robot [79,80] (see Figure 15a). The gripper was tested with 20 artificial aubergines and 25 pieces of real fruits ( 5 pieces of sweet peppers, pears, lemons, tomatoes and kiwis, respectively) that had reached a stage of sufficient maturity to be edible and be satisfactorily accepted by consumers. Figure $15 \mathrm{~b}$ displays an example sequence of the harvesting process. The experimental results show that the proposed design is capable of ensuring sufficient contact areas that provide stable grips for different types of fruits, even when the target is not centered on the midpoint of the gripper during the grasping (see Figure 15c). In this last particular case, the grasping is carried out by means of only two actuators. The actual harvested fruits (see Figure 15d) were also carefully observed both after the grasping tests and $24 \mathrm{~h}$ later, and no surface damage was detected.

Table 3 summarizes the main characteristics obtained experimentally from both a single soft gripper module and a hexagonal gripper endowed with six soft actuators.

Finally, Figure 16 lists the preferred soft gripper configurations for the different types of fruits. This classification was made taking into account: (i) the particular characteristics of the fruits to be harvested, such as their dimensions, mass, orientation and shape; and (ii) the intrinsic properties of the proposed gripper configurations, including the gripping range (maximum diameter between two contact points), the maximum lifting mass and the number of contact points.

Table 3. Characterization of a soft gripper module and a hexagonal configuration gripper endowed with six soft actuators.

\begin{tabular}{cc}
\hline Characteristic & Value \\
\hline Mass of a single module fully mounted & $69 \times 10^{-3} \mathrm{~kg}$ \\
Max. displacement of the soft actuator $(75 \mathrm{kPa})$ & $0.017 \mathrm{~m}$ \\
Max. contact force $(75 \mathrm{kPa})$ & $54 \mathrm{~N}$ \\
Operating pressure range & $0-75 \mathrm{kPa}$ \\
Slip payload test $(50 \mathrm{kPa})$ & $0.3 \mathrm{~kg}$ \\
Mean response time & $10 \mathrm{~kg}$ \\
\hline
\end{tabular}




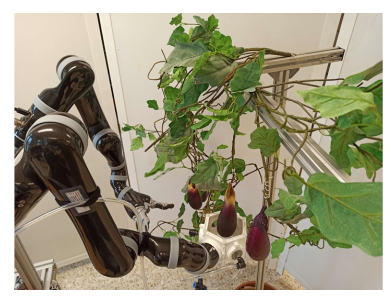

(a)
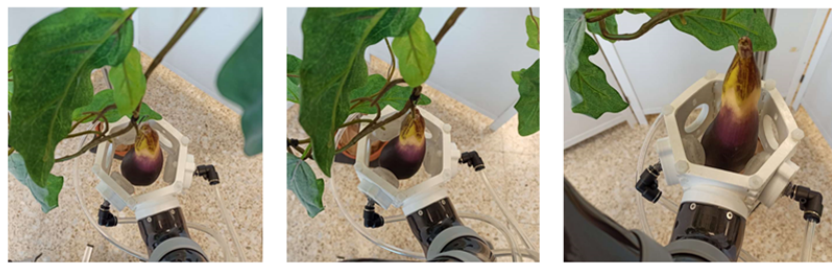

(b)
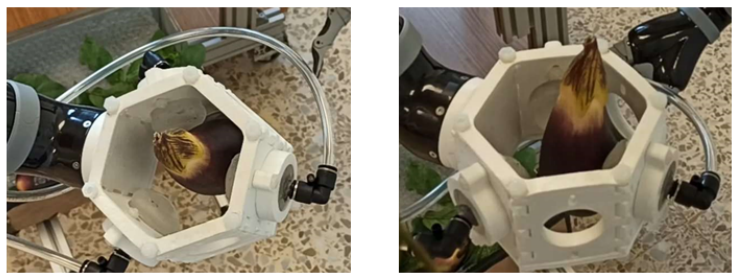

(c)
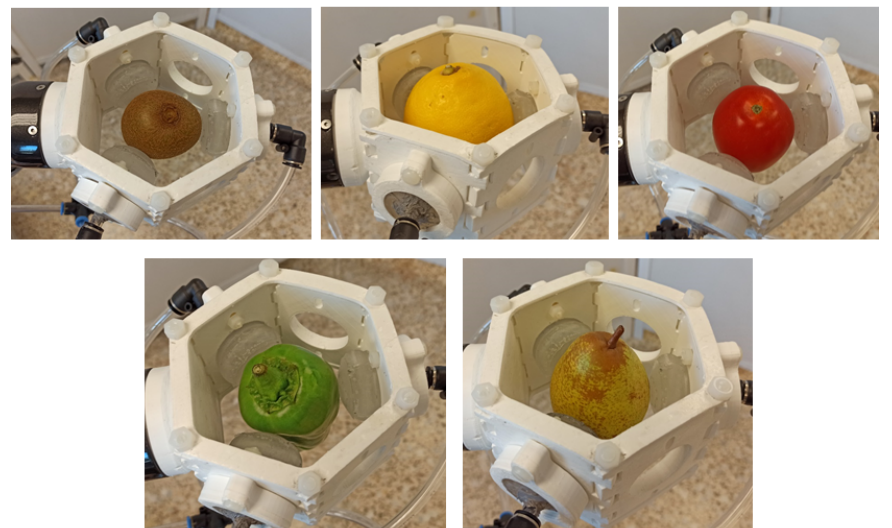

(d)

Figure 15. Experimental tests to evaluate the grasping performance. (a) Soft gripper with hexagonal configuration mounted on one of the Kinova Mico manipulators that make up the ROBOCROP dual-arm robot. (b) Example sequence of the harvesting process with the proposed soft gripper. (c) Grasping a target that is not centered on the midpoint of the gripper. (d) Evaluation of the soft gripper with several real fruits. 


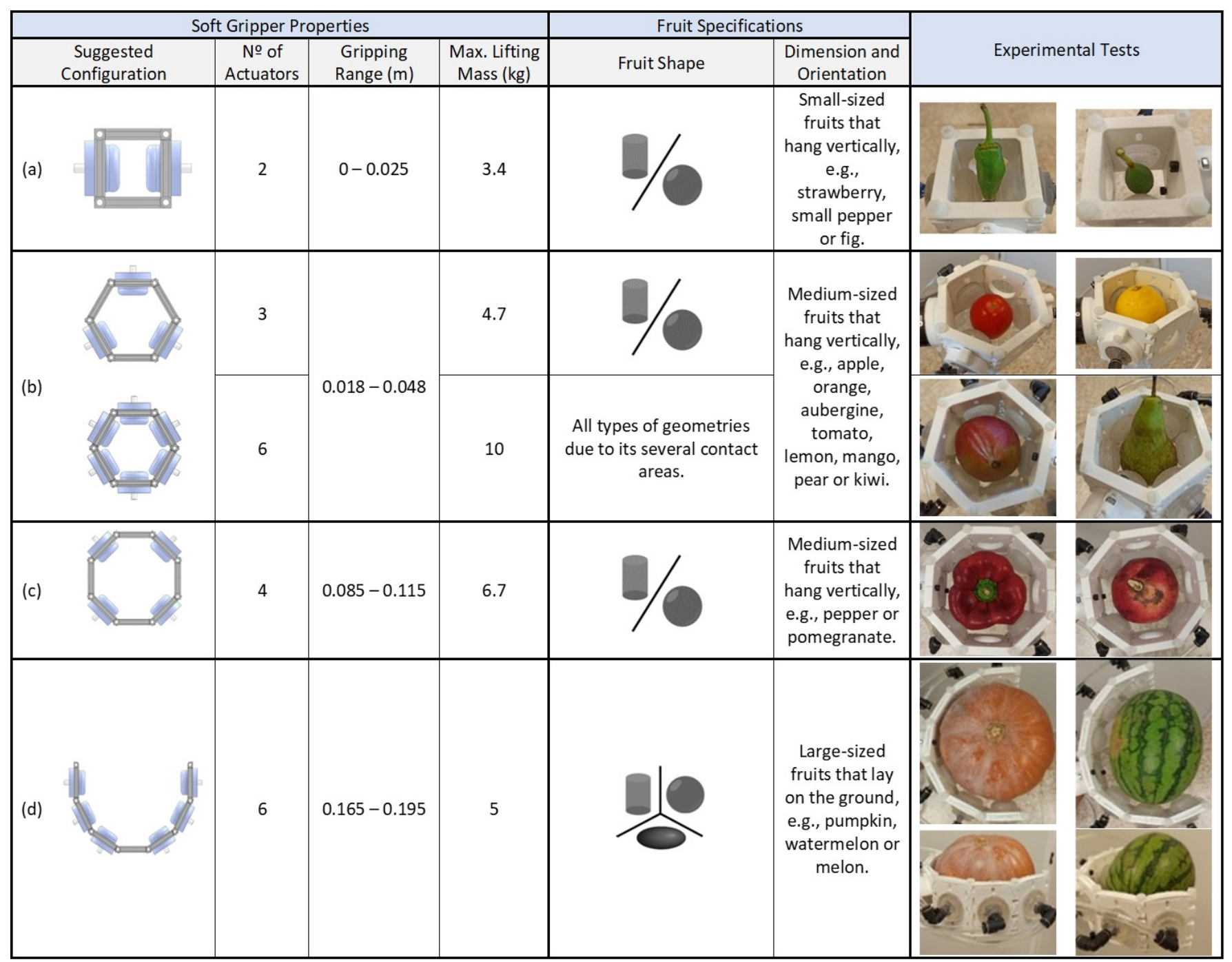

Figure 16. Preferred gripper configuration based on fruit type: (a) square configuration (two soft actuators); (b) hexagonal configuration (three and six soft actuators); (c) octagonal configuration (four soft actuators); and (d) open configuration (six soft actuators).

As can be seen in Figure 16, the square configuration is suitable for small fruits. In this case, the gripper was tested with a small pepper of $11.6 \times 10^{-3}$ and a fig of $7.1 \times 10^{-3} \mathrm{~kg}$. On the other hand, the hexagonal configuration equipped with three soft actuators is capable of gripping a wide variety of fruits, given its grasping range and load capacity. However, the hexagonal configuration with six soft actuators is the most recommended to ensure the grip on heavy non-symmetrical fruits, since this gripper is capable of lifting more weight than that of three actuators, due to its multitude of contact areas. In the examples presented in Figure 16, the hexagonal configuration gripper equipped with three soft actuators was used to grasp a tomato of $0.11 \mathrm{~kg}$ and a lemon of $96.6 \times 10^{-3} \mathrm{~kg}$, while the hexagonal configuration gripper equipped with six soft actuators was used to grasp a mango of $0.38 \mathrm{~kg}$ and a pear of $0.20 \mathrm{~kg}$. The octagonal configuration, such as the previous one, is also versatile, since it can provide two, three or four contact areas, being suitable for most medium-sized fruits. The gripper was tested with a pomegranate and a red bell pepper of 0.49 and $0.33 \mathrm{~kg}$, respectively. Lastly, the open configuration is indicated for medium-to-large fruits that usually lay on the grown. It is important to note that for this configuration it is necessary to adapt the pressure of each actuator independently, since generally the actuators at the ends support the load of the target, while those on the inside help to prevent its rotation during the grasping. This configuration was experimentally tested with a pumpkin and a watermelon of 2.26 and $2.15 \mathrm{~kg}$, respectively. 


\section{Conclusions}

The soft robotics field has been rapidly increasing, especially in sectors such as manipulation and rehabilitation. However, less attention has been given to the agricultural sector, where soft technology can have a significant impact, enabling the development of robotic harvesters with responsive, safe and adaptable grasping capabilities.

This article proposes a modular soft gripper design whose main features are its versatility, ease of manufacture and assembly, affordability and adaptability for medium- to large-sized fruits and the capability to handle agricultural products with all the advantages offered by soft robotics technology. To this end, the concept of diaphragm-type soft actuators, particularly bellows-type design, is studied in depth by analyzing and experimentally checking their behavior.

Furthermore, several steps were proposed to quantitatively determine the characteristics of the soft actuators, an often controversial issue, covered quite briefly in the literature, especially with regard to the tools used to perform such measurements. The relevance of soft characterization is crucial for its implementation in the industry and in other sectors, such as agriculture and health, to make a fair benchmark. For this reason, the proposed soft grippers are characterized in a broad sense, and the measuring instruments used are also presented.

As a future line, it is intended to characterize sensorization, as well as to integrate higher-level controllers based on artificial intelligence and machine learning, using the virtues of diaphragm-type soft actuators. The trajectory planning of the ROBOCROP dualarm robot endowed with the proposed soft gripper for harvesting applications will also be investigated. Furthermore, the energy consumption in this type of soft actuator $[81,82]$ could also be examined to increase its autonomy in real agricultural applications $[38,83-85]$. The study of methods to accurately assess the extent of surface and internal fruit damage caused by excessive external forces from this type of gripper will also be addressed. Finally, we will also carry out durability tests that analyze the life cycle of the proposed soft actuators during the continuous operation of the grippers.

Author Contributions: Conceptualization, E.N. and R.F.; methodology, E.N. and P.G.-d.-S.; validation, M.A.; formal analysis E.N.; investigation, E.N.; writing-original draft preparation, E.N.; writing-review and editing R.F.; visualization, E.N.; supervision R.F.; funding acquisition R.F. and P.G.-d.-S. All authors have read and agreed to the published version of the manuscript.

Funding: The research leading to these results has received funding from (i) FEDER/Ministerio de Ciencia, Innovación y Universidades-Agencia Estatal de Investigación/Proyecto ROBOCROP (DPI201784253-C2-1-R), (ii) RoboCity2030-DIH-CM, Madrid Robotics Digital Innovation Hub, S2018/NMT-4331, funded by "Programas de Actividades I+D en la Comunidad de Madrid" and cofunded by Structural Funds of the EU, and (iii) CSIC under Grant 202050E099, Proyecto Intramural IAMC-ROBI (Inteligencia Artificial y Mecatrónica Cognitiva para la Manipulación Robótica Bimanual).

Institutional Review Board Statement: Not applicable.

Informed Consent Statement: Not applicable.

Data Availability Statement: Not applicable.

Conflicts of Interest: The authors declare no conflict of interest.

\section{References}

1. Giannaccini, M.E.; Georgilas, I.; Horsfield, I.; Peiris, B.; Lenz, A.; Pipe, A.G.; Dogramadzi, S. A variable compliance, soft gripper. Auton. Robot. 2014, 36, 93-107. [CrossRef]

2. Yap, H.K.; Lim, J.H.; Goh, J.C.H.; Yeow, C.H. Design of a Soft Robotic Glove for Hand Rehabilitation of Stroke Patients With Clenched Fist Deformity Using Inflatable Plastic Actuators. J. Med. Devices 2016, 10, 1932-6181. [CrossRef]

3. Burgner-Kahrs, J.; Rucker, D.C.; Choset, H. Continuum Robots for Medical Applications: A Survey. IEEE Trans. Robot. 2015, 31, 1261-1280. [CrossRef]

4. Polygerinos, P.; Wang, Z.; Galloway, K.C.; Wood, R.J.; Walsh, C.J. Soft robotic glove for combined assistance and at-home rehabilitation. Robot. Auton. Syst. 2015, 73, 135-143. [CrossRef] 
5. Ansari, Y.; Falotico, E.; Mollard, Y.; Busch, B.; Cianchetti, M.; Laschi, C. A Multiagent Reinforcement Learning approach for inverse kinematics of high dimensional manipulators with precision positioning. In Proceedings of the 2016 6th IEEE International Conference on Biomedical Robotics and Biomechatronics (BioRob), Singapore, 26-29 June 2016; pp. 457-463. [CrossRef]

6. Goldfield, E.C.; Park, Y.L.; Chen, B.R.; Hsu, W.H.; Young, D.; Wehner, M.; Kelty-Stephen, D.G.; Stirling, L.; Weinberg, M.; Newman, D.; et al. Bio-Inspired Design of Soft Robotic Assistive Devices: The Interface of Physics, Biology, and Behavior. Ecol. Psychol. 2012, 24, 300-327. [CrossRef]

7. Katzschmann, R.K.; DelPreto, J.; MacCurdy, R.; Rus, D. Exploration of underwater life with an acoustically controlled soft robotic fish. Sci. Robot. 2018, 3. [CrossRef] [PubMed]

8. Tolley, M.; Shepherd, R.; Mosadegh, B.; Galloway, K.; Wehner, M.; Karpelson, M.; Wood, R.; Whitesides, G. A Resilient, Untethered Soft Robot. Soft Robot. 2014, 1, 213-223. [CrossRef]

9. Chowdhary, G.; Gazzola, M.; Krishnan, G.; Soman, C.; Lovell, S. Soft Robotics as an Enabling Technology for Agroforestry Practice and Research. Sustainability 2019, 11, 6751. [CrossRef]

10. Anna, K. Shadow delivers first hand. Ind. Robot. Int. J. 2005, 32 , 15-16. [CrossRef]

11. Manti, M.; Hassan, T.; Passetti, G.; D’Elia, N.; Laschi, C.; Cianchetti, M. A bioinspired soft robotic gripper for adaptable and effective grasping. Soft Robot. 2015, 2, 107-116. [CrossRef]

12. De Barrie, D.; Margetts, R.; Goher, K. Simpa: Soft-grasp infant myoelectric prosthetic arm. IEEE Robot. Autom. Lett. 2020, 5, 698-703. [CrossRef]

13. Konishi, S.; Shimomura, S.; Tajima, S.; Tabata, Y. Implementation of soft microfingers for a hMSC aggregate manipulation system. Microsyst. Nanoeng. 2016, 2, 1-7. [CrossRef]

14. Renda, F.; Cianchetti, M.; Giorelli, M.; Arienti, A.; Laschi, C. A 3D steady-state model of a tendon-driven continuum soft manipulator inspired by the octopus arm. Bioinspir. Biomim. 2012, 7, 025006. [CrossRef]

15. McMahan, W.; Chitrakaran, V.; Csencsits, M.; Dawson, D.; Walker, I.D.; Jones, B.A.; Pritts, M.; Dienno, D.; Grissom, M.; Rahn, C.D. Field trials and testing of the OctArm continuum manipulator. In Proceedings of the 2006 IEEE International Conference on Robotics and Automation, ICRA 2006, Orlando, FL, USA, 15-19 May 2006; pp. 2336-2341.

16. Marchese, A.D.; Onal, C.D.; Rus, D. Autonomous soft robotic fish capable of escape maneuvers using fluidic elastomer actuators. Soft Robot. 2014, 1, 75-87. [CrossRef]

17. Calisti, M.; Arienti, A.; Giannaccini, M.E.; Follador, M.; Giorelli, M.; Cianchetti, M.; Mazzolai, B.; Laschi, C.; Dario, P. Study and fabrication of bioinspired octopus arm mockups tested on a multipurpose platform. In Proceedings of the 2010 3rd IEEE RAS \& EMBS International Conference on Biomedical Robotics and Biomechatronics, Tokyo, Japan, 26-29 September 2010; pp. 461-466.

18. Manti, M.; Cacucciolo, V.; Cianchetti, M. Stiffening in soft robotics: A review of the state of the art. IEEE Robot. Autom. Mag. 2016, 23, 93-106. [CrossRef]

19. Duckett, T.; Pearson, S.; Blackmore, S.; Grieve, B.; Chen, W.H.; Cielniak, G.; Cleaversmith, J.; Dai, J.; Davis, S.; Fox, C.; et al. Agricultural robotics: The future of robotic agriculture. arXiv 2018, arXiv:1806.06762.

20. Li, Z.; Miao, F.; Yang, Z.; Wang, H. An anthropometric study for the anthropomorphic design of tomato-harvesting robots. Comput. Electron. Agric. 2019, 163, 104881. [CrossRef]

21. Yaguchi, H.; Nagahama, K.; Hasegawa, T.; Inaba, M. Development of an autonomous tomato harvesting robot with rotational plucking gripper. In Proceedings of the 2016 IEEE/RSJ International Conference on Intelligent Robots and Systems (IROS), Daejeon, Korea, 9-14 October 2016; pp. 652-657.

22. Mu, L.; Cui, G.; Liu, Y.; Cui, Y.; Fu, L.; Gejima, Y. Design and simulation of an integrated end-effector for picking kiwifruit by robot. Inf. Process. Agric. 2020, 7, 58-71. [CrossRef]

23. Huang, Z.; Sklar, E.; Parsons, S. Design of automatic strawberry harvest robot suitable in complex environments. In Proceedings of the Companion of the 2020 ACM/IEEE International Conference on Human-Robot Interaction, Cambridge, UK, 23-26 March 2020; pp. 567-569.

24. United Nations Department of Economic and Social Affairs. United Nations Department of Economic and Social Affairs, Population Division. World Population Prospects: The 2015 Revision, Key Findings and Advance Tables; Technical Report; Working Paper No. ESA/P/WP. 241; United Nations Department of Economic and Social Affairs: New York, NY, USA, 2015.

25. Vu, Q.; Kuzov, M.; Ronzhin, A. Hierarchical classification of robotic grippers applied for agricultural object manipulations. In Proceedings of the MATEC Web of Conferences, St. Petersburg, Russia, 18-29 April 2018; Volume 161, p. 03015.

26. Gonzalez-de-Santos, P.; Fernández, R.; Sepúlveda, D.; Navas, E.; Emmi, L.; Armada, M. Field Robots for Intelligent Farms-Inhering Features from Industry. Agronomy 2020, 10, 1638. [CrossRef]

27. Font, D.; Pallejà, T.; Tresanchez, M.; Runcan, D.; Moreno, J.; Martínez, D.; Teixidó, M.; Palacín, J. A proposal for automatic fruit harvesting by combining a low cost stereovision camera and a robotic arm. Sensors 2014, 14, 11557-11579. [CrossRef] [PubMed]

28. Salinas, C.; Fernández, R.; Montes, H.; Armada, M. A New Approach for Combining Time-of-Flight and RGB Cameras Based on Depth-Dependent Planar Projective Transformations. Sensors 2015, 15, 24615-24643. [CrossRef]

29. Fernández, R.; Montes, H.; Salinas, C. VIS-NIR, SWIR and LWIR Imagery for Estimation of Ground Bearing Capacity. Sensors 2015, 15, 13994-14015. [CrossRef]

30. Fernandez, R.; Montes, H.; Surdilovic, J.; Surdilovic, D.; Gonzalez-De-Santos, P.; Armada, M. Automatic detection of field-grown cucumbers for robotic harvesting. IEEE Access 2018, 6, 35512-35527. [CrossRef] 
31. Agostini, A.; Alenya, G.; Fischbach, A.; Scharr, H.; Woergoetter, F.; Torras, C. A cognitive architecture for automatic gardening. Comput. Electron. Agric. 2017, 138, 69-79. [CrossRef]

32. Zion, B.; Mann, M.; Levin, D.; Shilo, A.; Rubinstein, D.; Shmulevich, I. Harvest-order planning for a multiarm robotic harvester. Comput. Electron. Agric. 2014, 103, 75-81. [CrossRef]

33. Masuzawa, H.; Miura, J.; Oishi, S. Development of a mobile robot for harvest support in greenhouse horticulture-Person following and mapping. In Proceedings of the 2017 IEEE/SICE International Symposium on System Integration (SII), Taipei, Taiwan, 11-14 December 2017; pp. 541-546. [CrossRef]

34. Boyraz, P.; Runge, G.; Raatz, A. An overview of novel actuators for soft robotics. Actuators 2018, 7, 48. [CrossRef]

35. Shintake, J.; Cacucciolo, V.; Floreano, D.; Shea, H. Soft robotic grippers. Adv. Mater. 2018, 30, 1707035. [CrossRef]

36. Navas, E.; Fernández, R.; Sepúlveda, D.; Armada, M.; Gonzalez-de Santos, P. Soft Grippers for Automatic Crop Harvesting: A Review. Sensors 2021, 21, 2689. [CrossRef] [PubMed]

37. Falco, J.; Van Wyk, K.; Liu, S.; Carpin, S. Grasping the performance: Facilitating replicable performance measures via benchmarking and standardized methodologies. IEEE Robot. Autom. Mag. 2015, 22, 125-136. [CrossRef]

38. Navas, E.; Fernandez, R.; Sepúlveda, D.; Armada, M.; Gonzalez-de Santos, P. A Design Criterion Based on Shear Energy Consumption for Robotic Harvesting Tools. Agronomy 2020, 10, 734. [CrossRef]

39. Sepúlveda, D.; Fernández, R.; Navas, E.; González-de Santos, P.; Armada, M. ROS framework for perception and dual-arm manipulation in unstructured environments. In Iberian Robotics Conference; Springer: Cham, Switzerland, $2019 ;$ pp. $137-147$.

40. Navas, E.; Fernández, R.; Sepúlveda, D.; Armada, M.; Gonzalez-de Santos, P. Soft Gripper for Robotic Harvesting in Precision Agriculture Applications. In Proceedings of the 2021 IEEE International Conference on Autonomous Robot Systems and Competitions (ICARSC), Santa Maria da Feira, Portugal, 28-29 April 2021; pp. 167-172.

41. Hsiao, L.Y.; Jing, L.; Li, K.; Yang, H.; Li, Y.; Chen, P.Y. Carbon nanotube-integrated conductive hydrogels as multifunctional robotic skin. Carbon 2020, 161, 784-793. [CrossRef]

42. Mosadegh, B.; Polygerinos, P.; Keplinger, C.; Wennstedt, S.; Shepherd, R.F.; Gupta, U.; Shim, J.; Bertoldi, K.; Walsh, C.J.; Whitesides, G.M. Pneumatic networks for soft robotics that actuate rapidly. Adv. Funct. Mater. 2014, 24, 2163-2170. [CrossRef]

43. Wang, Z.; Or, K.; Hirai, S. A dual-mode soft gripper for food packaging. Robot. Auton. Syst. 2020, 125, 103427. [CrossRef]

44. Shih, B.; Drotman, D.; Christianson, C.; Huo, Z.; White, R.; Christensen, H.I.; Tolley, M.T. Custom soft robotic gripper sensor skins for haptic object visualization. In Proceedings of the 2017 IEEE/RSJ International Conference on Intelligent Robots and Systems (IROS), Vancouver, BC, Canada, 24-28 September 2017; pp. 494-501.

45. Connolly, F.; Walsh, C.J.; Bertoldi, K. Automatic design of fiber-reinforced soft actuators for trajectory matching. Proc. Natl. Acad. Sci. USA 2017, 114, 51-56. [CrossRef]

46. Seibel, A.; Yıldız, M.; Zorlubaş, B. A Gecko-Inspired Soft Passive Gripper. Biomimetics 2020, 5, 12. [CrossRef]

47. Teeple, C.B.; Koutros, T.N.; Graule, M.A.; Wood, R.J. Multi-segment soft robotic fingers enable robust precision grasping. Int. J. Robot. Res. 2020, 39, 1647-1667. [CrossRef]

48. Galloway, K.C.; Becker, K.P.; Phillips, B.; Kirby, J.; Licht, S.; Tchernov, D.; Wood, R.J.; Gruber, D.F. Soft robotic grippers for biological sampling on deep reefs. Soft Robot. 2016, 3, 23-33. [CrossRef]

49. Galley, A.; Knopf, G.K.; Kashkoush, M. Pneumatic Hyperelastic Actuators for Grasping Curved Organic Objects. Actuators 2019, 8, 76. [CrossRef]

50. Wang, W.; Rodrigue, H.; Kim, H.I.; Han, M.W.; Ahn, S.H. Soft composite hinge actuator and application to compliant robotic gripper. Compos. Part B Eng. 2016, 98, 397-405. [CrossRef]

51. Rodrigue, H.; Wang, W.; Kim, D.R.; Ahn, S.H. Curved shape memory alloy-based soft actuators and application to soft gripper. Compos. Struct. 2017, 176, 398-406. [CrossRef]

52. Modabberifar, M.; Spenko, M. A shape memory alloy-actuated gecko-inspired robotic gripper. Sens. Actuators A Phys. 2018, 276, 76-82. [CrossRef]

53. Johnston, I.; McCluskey, D.; Tan, C.; Tracey, M. Mechanical characterization of bulk Sylgard 184 for microfluidics and microengineering. J. Micromech. Microeng. 2014, 24, 035017. [CrossRef]

54. Wang, Z.; Xiang, C.; Yao, X.; Le Floch, P.; Mendez, J.; Suo, Z. Stretchable materials of high toughness and low hysteresis. Proc. Natl. Acad. Sci. USA 2019, 116, 5967-5972. [CrossRef] [PubMed]

55. Kim, T.K.; Kim, J.K.; Jeong, O.C. Measurement of nonlinear mechanical properties of PDMS elastomer. Microelectron. Eng. 2011, 88, 1982-1985. [CrossRef]

56. Ogden, R.W. Large deformation isotropic elasticity-on the correlation of theory and experiment for incompressible rubberlike solids. Proc. R. Soc. Lond. A Math. Phys. Sci. 1972, 326, 565-584. [CrossRef]

57. The future of plastic. Nat. Commun. 2018, 9, 1723-2041.

58. Krishnan, S.; Pandey, P.; Mohanty, S.; Nayak, S.K. Toughening of polylactic acid: An overview of research progress. Polymer-Plast. Technol. Eng. 2016, 55, 1623-1652. [CrossRef]

59. Odent, J.; Raquez, J.M.; Dubois, P. Highly toughened polylactide-based materials through melt-blending techniques. Biodegrad. Polyest. 2015, 235-274. [CrossRef]

60. Zeng, J.B.; Li, K.A.; Du, A.K. Compatibilization strategies in poly (lactic acid)-based blends. RSC Adv. 2015, 5, 32546-32565. [CrossRef] 
61. Kfoury, G.; Raquez, J.M.; Hassouna, F.; Odent, J.; Toniazzo, V.; Ruch, D.; Dubois, P. Recent advances in high performance poly (lactide): From "green" plasticization to super-tough materials via (reactive) compounding. Front. Chem. 2013, 1, 32. [CrossRef] [PubMed]

62. Liu, H.; Zhang, J. Research progress in toughening modification of poly (lactic acid). J. Polym. Sci. Part B Polym. Phys. 2011, 49, 1051-1083. [CrossRef]

63. Anderson, K.S.; Schreck, K.M.; Hillmyer, M.A. Toughening polylactide. Polym. Rev. 2008, 48, 85-108. [CrossRef]

64. Rasal, R.M.; Janorkar, A.V.; Hirt, D.E. Poly (lactic acid) modifications. Prog. Polym. Sci. 2010, 35, 338-356. [CrossRef]

65. Nagarajan, V.; Mohanty, A.K.; Misra, M. Perspective on polylactic acid (PLA) based sustainable materials for durable applications: Focus on toughness and heat resistance. ACS Sustain. Chem. Eng. 2016, 4, 2899-2916. [CrossRef]

66. Martinez, R.V.; Branch, J.L.; Fish, C.R.; Jin, L.; Shepherd, R.F.; Nunes, R.M.; Suo, Z.; Whitesides, G.M. Robotic tentacles with three-dimensional mobility based on flexible elastomers. Adv. Mater. 2013, 25, 205-212. [CrossRef] [PubMed]

67. Hao, Y.; Gong, Z.; Xie, Z.; Guan, S.; Yang, X.; Ren, Z.; Wang, T.; Wen, L. Universal soft pneumatic robotic gripper with variable effective length. In Proceedings of the 2016 35th Chinese Control Conference (CCC), Chengdu, China, 27-29 July 2016; pp. 6109-6114.

68. Zhou, J.; Chen, S.; Wang, Z. A soft-robotic gripper with enhanced object adaptation and grasping reliability. IEEE Robot. Autom. Lett. 2017, 2, 2287-2293. [CrossRef]

69. Friend, J.; Yeo, L. Fabrication of microfluidic devices using polydimethylsiloxane. Biomicrofluidics 2010, 4, 026502. [CrossRef]

70. Rateni, G.; Cianchetti, M.; Ciuti, G.; Menciassi, A.; Laschi, C. Design and development of a soft robotic gripper for manipulation in minimally invasive surgery: A proof of concept. Meccanica 2015, 50, 2855-2863. [CrossRef]

71. Linghu, C.; Zhang, S.; Wang, C.; Yu, K.; Li, C.; Zeng, Y.; Zhu, H.; Jin, X.; You, Z.; Song, J. Universal SMP gripper with massive and selective capabilities for multiscaled, arbitrarily shaped objects. Sci. Adv. 2020, 6, eaay5120. [CrossRef]

72. Rus, D.; Tolley, M.T. Design, fabrication and control of soft robots. Nature 2015, 521, 467-475. [CrossRef]

73. Duriez, C. Control of elastic soft robots based on real-time finite element method. In Proceedings of the 2013 IEEE International Conference on Robotics and Automation, Karlsruhe, Germany, 6-10 May 2013; pp. 3982-3987.

74. Park, W.; Seo, S.; Oh, J.; Bae, J. A Sensorized Hybrid Gripper to Evaluate a Grasping Quality Based on a Largest Minimum Wrench. IEEE Robot. Autom. Lett. 2020, 5, 3243-3250. [CrossRef]

75. McKenzie, R.M.; Barraclough, T.W.; Stokes, A.A. Integrating soft robotics with the robot operating system: A hybrid pick and place arm. Front. Robot. AI 2017, 4, 39. [CrossRef]

76. Xydas, N.; Kao, I. Modeling of contact mechanics and friction limit surfaces for soft fingers in robotics, with experimental results. Int. J. Robot. Res. 1999, 18, 941-950. [CrossRef]

77. Li, Y.; Kao, I. A review of modeling of soft-contact fingers and stiffness control for dextrous manipulation in robotics. In Proceedings of the Proceedings 2001 ICRA. IEEE International Conference on Robotics and Automation (Cat. No. 01CH37164), Seoul, Korea, 21-26 May 2001; Volume 3, pp. 3055-3060.

78. Robotics Company | Robotic Assistive Technology | Kinova. 2019. Available online: https://www.kinovarobotics.com (accessed on 29 August 2021).

79. Navas, E.; Fernández, R.; Sepúlveda, D.; Armada, M.; Gonzalez-de Santos, P. Modular dual-arm robot for precision harvesting. In Iberian Robotics Conference; Springer: Cham, Switzerland, 2019; pp. 148-158.

80. Sepúlveda, D.; Fernández, R.; Navas, E.; Armada, M.; González-De-Santos, P. Robotic aubergine harvesting using dual-arm manipulation. IEEE Access 2020, 8, 121889-121904. [CrossRef]

81. Krahn, J.M.; Fabbro, F.; Menon, C. A soft-touch gripper for grasping delicate objects. IEEE/ASME Trans. Mechatronics 2017, 22, 1276-1286. [CrossRef]

82. Wang, Y.; Gupta, U.; Parulekar, N.; Zhu, J. A soft gripper of fast speed and low energy consumption. Sci. China Technol. Sci. 2019, 62, 31-38. [CrossRef]

83. Igathinathane, C.; Pordesimo, L.; Schilling, M.; Columbus, E. Fast and simple measurement of cutting energy requirement of plant stalk and prediction model development. Ind. Crop. Prod. 2011, 33, 518-523. [CrossRef]

84. Visvanathan, R.; Sreenarayanan, V.; Swaminathan, K. Effect of knife angle and velocity on the energy required to cut cassava tubers. J. Agric. Eng. Res. 1996, 64, 99-102. [CrossRef]

85. Azadbakht, M.; Asl, A.R.; Zahedi, K.T. Energy requirement for cutting corn stalks (Single Cross 704 Var.). Int. J. Biol. Food Vet. Agric. Eng 2014, 8, 471-474. 